\title{
The effect of human resource management on performance in hospitals in Sub-Saharan Africa: a systematic literature review
}

\author{
Philipos Petros Gile ${ }^{1,2^{*}}$, Martina Buljac-Samardzic ${ }^{2}$ and Joris Van De Klundert ${ }^{2,3}$
}

\begin{abstract}
Hospitals in Sub-Saharan Africa (SSA) face major workforce challenges while having to deal with extraordinary high burdens of disease. The effectiveness of human resource management (HRM) is therefore of particular interest for these SSA hospitals. While, in general, the relationship between HRM and hospital performance is extensively investigated, most of the underlying empirical evidence is from western countries and may have limited validity in SSA. Evidence on this relationship for SSA hospitals is scarce and scattered. We present a systematic review of empirical studies investigating the relationship between HRM and performance in SSA hospitals.

Following the PRISMA protocol, searching in seven databases (i.e., Embase, MEDLINE, Web of Science, Cochrane, PubMed, CINAHL, Google Scholar) yielded 2252 hits and a total of 111 included studies that represent 19 out of 48 SSA countries. From a HRM perspective, most studies researched HRM bundles that combined practices from motivation-enhancing, skills-enhancing, and empowerment-enhancing domains. Motivation-enhancing practices were most frequently researched, followed by skills-enhancing practices and empowerment-enhancing practices. Few studies focused on single HRM practices (instead of bundles). Training and education were the most researched single practices, followed by task shifting. From a performance perspective, our review reveals that employee outcomes and organizational outcomes are frequently researched, whereas team outcomes and patient outcomes are significantly less researched. Most studies report HRM interventions to have positively impacted performance in one way or another. As researchers have studied a wide variety of (bundled) interventions and outcomes, our analysis does not allow to present a structured set of effective one-to-one relationships between specific HRM interventions and performance measures. Instead, we find that specific outcome improvements can be accomplished by different HRM interventions and conversely that similar HRM interventions are reported to affect different outcome measures.

In view of the high burden of disease, our review identified remarkable little evidence on the relationship between HRM and patient outcomes. Moreover, the presented evidence often fails to provide contextual characteristics which are likely to induce variety in the performance effects of HRM interventions. Coordinated research efforts to advance the evidence base are called for.
\end{abstract}

Keywords: Systematic review, HRM, SSA, Hospital, Performance, Outcomes, Health workforce

\footnotetext{
* Correspondence: gile@eshpm.eur.nl

'Higher Education Institutions' Partnership, PO BOX 14051, Addis Ababa,

Ethiopia

${ }^{2}$ Erasmus School of Health Policy \& Management, Erasmus University

Rotterdam, PO Box 1738, 3000 DR Rotterdam, The Netherlands

Full list of author information is available at the end of the article
}

(c) The Author(s). 2018 Open Access This article is distributed under the terms of the Creative Commons Attribution 4.0 International License (http://creativecommons.org/licenses/by/4.0/), which permits unrestricted use, distribution, and reproduction in any medium, provided you give appropriate credit to the original author(s) and the source, provide a link to the Creative Commons license, and indicate if changes were made. The Creative Commons Public Domain Dedication waiver (http://creativecommons.org/publicdomain/zero/1.0/) applies to the data made available in this article, unless otherwise stated. 


\section{Background}

While Sub-Saharan Africa (SSA) is home to $12 \%$ of the global population [1], it employs $3.5 \%$ of the global health workforce to service a disproportionate $27 \%$ of the global burden of disease [2]. A majority of countries across the globe for which the health workforce shortage is classified as critical (36 out of 57) lie in SSA [3, 4]. Most SSA countries are not able to attain an average health workforce density of 2.5 per 1000 population as recommended by the World Health Organization (WHO) [5, 6] and half of the SSA countries have fewer than ten physicians per 100,000 people (while Western countries commonly have 250 per 100,000 or more) [ 5 , 7-9]. The low workforce density and high workload in SSA especially impacts hospital $[6,7]$. The shortage of supply to match demand further increases because of low retention rates among skilled health workers [8-12]. Implementation of human resource management (HRM) practices is needed to improve the situation for a depleted and overstretched health workforce, and patient outcomes [10, 13-18].

Research on HRM interventions in SSA hospitals have so far primarily addressed (human) resource availability, e.g., "head counts," technical skills, and basic working conditions [19-28]. These practices are often referred to as "hard" HRM [29]. Hard HRM refers to approaching employees as one of several categories of organization resources (e.g., financial resources, equipment) that influence organizational effectiveness and are mostly organization-centered and reactive [26, 29, 30]. Although hard HRM practices have shown to be related to improved performance outcomes (e.g., waiting time, quality of care, patient experiences) [18, 31, 32], broader HRM interventions are needed to sustain hospital service quality and retain a satisfied workforce [10, 24].

Soft HRM practices are more employee-centered and focused on work-environment. They single out human resources as most important and subsequently address training and development needs, tasks and roles, communication, delegation, and motivation [29, 33, 34]. In the last decade, especially soft HRM practices have shown to impact performance, sometimes in combination with hard HRM practices [25, 33, 35]. However, understanding and the adoption of soft HRM practices in SSA hospitals is limited [18, 36-38].

The growing evidence of the relationship between HRM practices and performance has shown to be complex and is frequently referred to as "black box" [39-42]. Dieleman et al. underline the importance of context when stating that a HRM practice may result in different outcomes when applied in different contexts, as contextual factors are likely to influence outcomes [16].

The current evidence base on effectiveness of HRM practices is mainly developed in particular research settings, namely hospitals in the USA and Western Europe. Next to the high variation within these settings (e.g., type of hospital, financial management, government), there are major differences compared to the SSA setting (e.g., low providers capacity, low economic status, challenging socio-cultural issues, demographic trends, high disease burden). It is therefore likely to have limited validity in SSA [34]. A first relevant and major contextual difference is formed by the combination of a disproportionally high burden of disease and health workforce shortages occurring in SSA contexts, which so explicitly outline the societal relevance of understanding the relationship between HRM practices and performance [43-46]. In addition, major cultural differences exist, as well as differences in public service infrastructures and operations [36], financial resource limitations, availability and quality of medicines, materials and equipment, disease prevalence, and health literacy [10, 34, 37, 47-52]. Rowe et al. highlighted the need to generate knowledge about the strategies to improve performance by HRM practices in low-resource settings and called for dedicated and updated systematic reviews [18]. Harries and Salaniponi underlined this by stating that "getting the most out of the already depleted and overstretched health workforce in resource-poor areas is a priority" [52]. This study presents a systematic literature review on the relationship between HRM and performance for SSA hospitals.

\section{Methods}

We conducted this systematic literature review following the Preferred Reporting Items for Systematic Reviews and Meta-Analyses (PRISMA) [53-55].

\section{Search strategy}

The search included seven databases (see Table 1) with search terms from three categories:

1. The geographical SSA setting as defined by United Nations [56]. For example, terms regarding SSA or

Table 1 Number of hits per database

\begin{tabular}{ll}
\hline Database & $\begin{array}{l}\text { Number } \\
\text { of hits }\end{array}$ \\
\hline Embase & 1217 \\
MEDLINE & 355 \\
Web of Science & 186 \\
Cochrane & 1 \\
PubMed & 49 \\
CINAHL & 286 \\
Google Scholar & 157 \\
Total & 2251 \\
\hline
\end{tabular}


the SSA countries separately (e.g., Benin, Ethiopia, Kenya, South Africa).

2. Healthcare setting and healthcare workforce. For example, hospitals or physician.

3. Terminologies related to HRM practices. For example, human resource management, training, skills, motivation, competences, or compensation.

Additional file 1 provides search term details. The search strategy was conducted in collaboration with a librarian from a medical library specialized in designing systematic reviews in April 2016. The search strategy resulted in 2251 titles/abstracts (doubles excluded) (see Table 1).

\section{Inclusion/exclusion criteria}

Studies were included if they met the following inclusion criteria: (1) Empirical study, regardless of the research methods; (2) focusing on links between HRM and performance outcomes; (3) SSA region; (4) hospital setting; (5) English language; and (6) published in a peer reviewed scientific journal.

Studies were excluded based on the following exclusion criteria: (1) focus on technical skills only (e.g., clinical skills training) as opposed to non-technical skills (e.g., team work training, personal communication training) $[57,58]$; (2) HRM interventions which were not under the control of hospital management but enforced by the Ministry of Health or external partner organizations such as the WHO (e.g., a national HIV educational intervention); and (3) studies that solely address capacity shortage (e.g., employing additional nurses). Studies which solely report on reducing capacity shortages are excluded as they are expected to improve effectiveness by definition.

\section{Selection strategy}

(1) We followed a four-stage selection process using a structured Excel format [59]: screening the title and abstract on the in- and exclusion criteria. This was performed independently by two authors. In case of disagreement between the two authors, the third author decided or postponed the decision to the next stage. The first stage reduced the initial search of 2251 hits to 409 hits.

(2) Examining the full text on the in- and exclusion criteria. The second stage was also performed by two authors. In case of disagreement, the third author was included to make the final decision. The second stage reduced the publications to 110 articles.

(3) Summarizing all accepted full articles by the first author.
(4) Reference and biography check of the summarized articles resulted in including one additional article and hence a total of 111 included articles (see Fig. 1).

\section{Data analysis}

The first data analysis step was to collect all HRM practice and all performance outcomes from the included studies. These "raw" practices and outcomes were discussed within the research team and processed iteratively to determine common "labels" for the practices and outcomes. These labels practices and outcomes where subsequently structured in categories. Building on previous syntheses in HRM effectiveness research $[19,20,27,60]$, we distinguished five categories of (single) HRM practices (see Table 5):

(1) Training and education;

(2) Salary and compensation;

(3) Rostering and scheduling;

(4) Task shifting; and

(5) Managing employees (through leadership support and mentoring).

All labeled practices from the data collection process on single HRM practices were categorized accordingly. Additional file 2 presents the number of studies that link a specific HRM practice to a specific outcome. Studies presenting research on HRM bundles, i.e., interventions which combine multiple practices, are classified following Subramony ([28], p. 746-747]) (see Table 2). The five categories of single HRM practices can be placed under the classification of Subramony as follows: empowerment enhancing (task shifting), motivation enhancing (salary and compensation, rostering and scheduling, managing employees), and skills enhancing (training and education).

The performance outcome dimensions were categorized into four categories:

(1) Employee outcomes (employee performance, job satisfaction, turnover intention or retention, motivation, workload reduction, reduction of moonlighting);

(2) Team performance outcome;

(3) Organizational outcomes (quality of care, waiting time, efficiency, patient safety/error reduction, staff shortage reduction); and

(4) Patient outcomes (patient experience, clinical outcome).

\section{Quality appraisal}

We appraised the quality of the studies using the revised version (2011) of the Mixed Methods Appraisal Tool (MMAT) [61-63], as commonly applied in systematic reviews (e.g., [64-67]). For qualitative and quantitative 


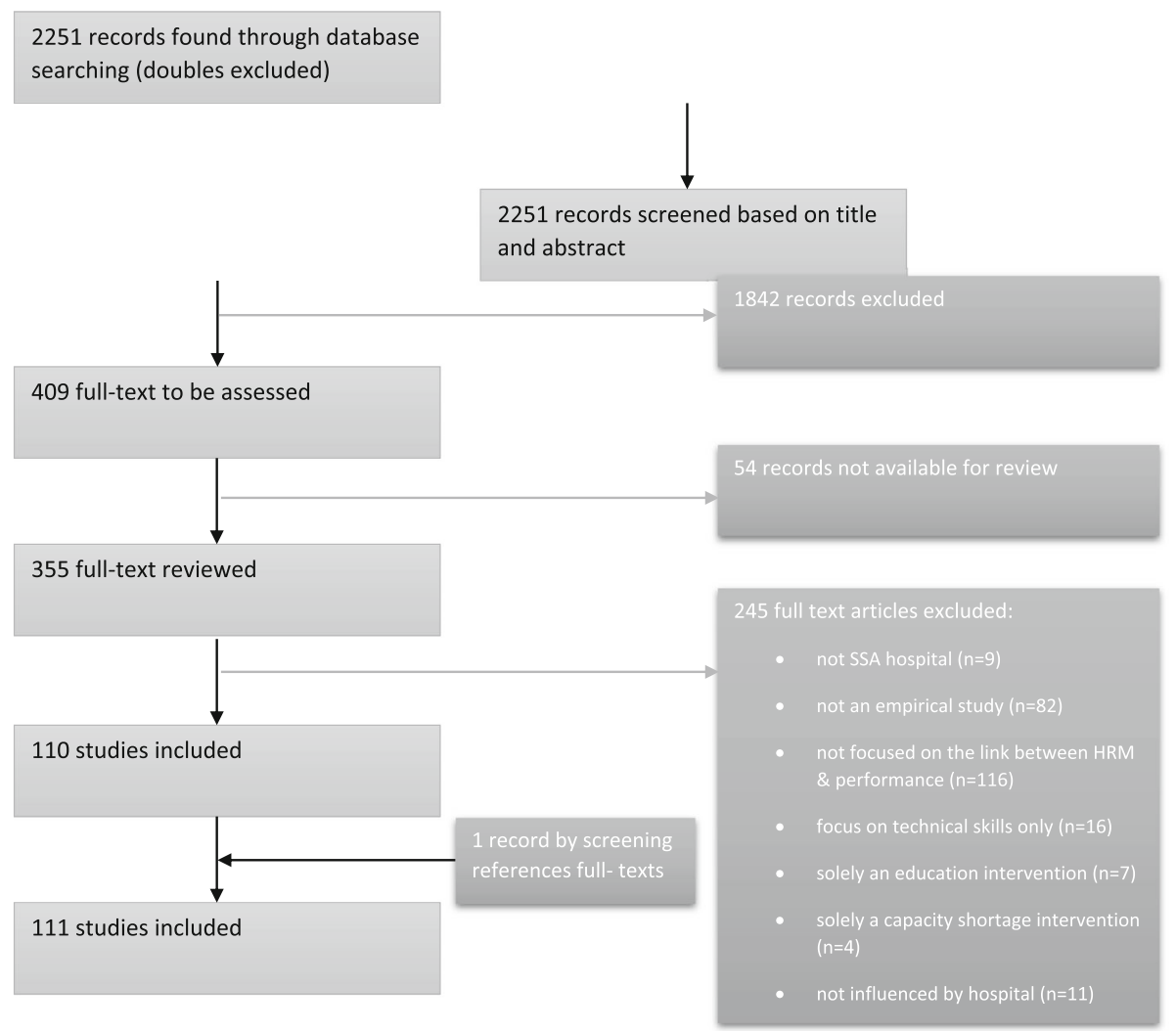

Fig. 1 PRISMA Flow Diagram

studies, the scores represent the number of criteria met, varying from one criterion met $\left(^{*}\right)$ to all criteria met $(* * * *)$. For mixed method studies, the scores represent the lowest score of the quantitative and qualitative components, as the quality of the study cannot surpass the quality of its weakest component. Tables 5 and 6 present the MMAT scores of the included studies.

Table 2 Content of HRM bundles according to Subramony (2009)

Empowerment-enhancing bundles

Employee involvement in influencing work process/outcomes

Formal grievance procedure and complaint resolution systems

Job enrichment (skill flexibility, job variety, responsibility)

Self-managed or autonomous work groups

Employee participation in decision making

Systems to encourage feedback from employees

Motivation-enhancing bundles

Formal performance appraisal process

Incentive plans (bonuses, profit-sharing, gain-sharing plans)

Linking pay to performance

Opportunities for internal career mobility and promotions

Health care and other employee benefits

Skills-enhancing bundles

Job descriptions/requirements generated through job analysis

Job-based skill training

Recruiting to ensure availability of large applicant pools

Structured and validated tools/procedures for personnel selection

\section{Results}

\section{Study characteristics}

The selected studies represent 19 out of 48 SSA countries (presented in Additional file 3). The six most studied countries are South Africa (32 studies), Tanzania (14), Kenya (13), Nigeria (10), Ethiopia (8), and Uganda (8). Five studies researched hospitals in multiple SSA countries. As a research setting, 16 studies simply mention hospitals without specifying the type of hospital, in contrast to the others that specified whether it regarded public, national, private, missionary, teaching, district, secondary care, rural, and/or primary care hospitals. The research included 36 qualitative (32.4\%), 57 quantitative (51.3\%), and 18 mixed methods (16.2\%) studies. Table 3 displays the MMAT quality scores of the included studies.

\section{Link between HRM practices and performance outcomes}

Table 4 shows that while most studies $(n=85,76.6 \%)$ considered a bundle of HRM interventions (as opposed to a single practice intervention), they typically addressed only one performance outcome $(n=81,73.0 \%)$. For ease of exposition, we now first present a narrative synthesis of the results on single HRM practices and subsequently of the results on HRM bundles. Table 5 
Table 3 Number of studies with study design and methodological appraisal scores

\begin{tabular}{llllll}
\hline MMAT score & & & & & \\
\hline Study design & $25 \%$ & $50 \%$ & $75 \%$ & $100 \%$ & Total \\
& $*$ & $* *$ & $* * *$ & $* * * *$ & \\
\hline Quantitative & - & 13 & 18 & 26 & 57 \\
Qualitative & 1 & 8 & 13 & 14 & 36 \\
Mixed methods & 1 & 3 & 9 & 5 & 18 \\
Total & 2 & 24 & 40 & 45 & 111 \\
\hline
\end{tabular}

(single HRM practices) and Table 6 (HRM bundles) present detailed review findings and the corresponding references.

\section{Single HRM practices and performance outcomes}

In total 18 single HRM practices were researched (see Additional file 2). The single HRM practices are clustered in five categories:

(1) Training and education Ten studies presented evidence on the relationship between training and outcomes [75, 94, 100, 102, 148, 150, 155, 146, 166, 172]. Six of these studies considered employee outcomes, two of which found a positive association with job satisfaction and retention [166, 172]. Four other studies (from South Africa, Tanzania, and Nigeria) [94, 102, 146, 148] found negative relationships between training and employee outcomes. Two of these studies also reported improved team performance as a result of team-training, but their evidence was qualified as weak $[146,172]$. Three of the four studies focusing on organizational outcomes reported improvements in the quality of care [94, 150, 166]. The two studies reporting on patient outcomes found non-significant reductions in (maternal) mortality rates $[155,172]$.

(2)Salary and compensation Research on salary and compensation almost exclusively regarded individual employee level outcomes (four studies). More specifically, they reported employee performance improvement $[69,83,120]$, and one study reported improved employee retention [80].

(3)Rostering and scheduling The four studies on rostering and scheduling each reported different, yet

Table 4 Number of performance outcomes for HRM practices

\begin{tabular}{lllll}
\hline HRM practices & \multicolumn{2}{l}{ Number of performance outcomes } & Total \\
\cline { 2 - 4 } & 1 outcome & 2 outcomes & 3 outcomes & \\
\hline Single HRM practice & 19 & 7 & 0 & 26 \\
Bundles of HRM practices & 62 & 22 & 1 & 85 \\
Total number of studies & 81 & 29 & 1 & 111 \\
\hline
\end{tabular}

positive, effects on employee outcomes or organizational outcomes [121, 122, 126, 154]. One low-quality study [154] reported failure of HRM interventions (e.g., staff control strategies and scheduling/rostering) to reduce turnover intention.

(4)Task shifting The six studies that researched task shifting/task delegation reported organizational outcomes. Three of the studies reported improvement in efficiency, while the other three reported to have reduced employee shortages. Interestingly, the evidence reported on the relationship with clinical outcome and quality of care was inconclusive (e.g., $[95,96])$.

(5)Managing employees through leadership support and mentoring The two studies which involved leadership and mentoring practices both reported improved job satisfaction by employees $[158,160]$.

\section{HRM bundles and performance outcomes}

Table 6 shows that the majority of the studies that researched HRM bundles have considered bundles that combine practices from multiple HRM themes (i.e., empowerment, motivation, and skill).

\section{Motivation-enhancing HRM practices}

Motivation-enhancing practices $(n=71,83.5 \%)$ are the most researched in SSA and refer to intrinsic and/or extrinsic motivation-enhancing HRM practices in a bundle. Five studies (6\%) considered bundles that only included motivation-enhancing practices [88, 97, 123, 125, 156]. These studies reported improved employee outcomes, such as job satisfaction, performance, retention, and staff motivation.

Many studies reported on bundles combining motivation- and skills-enhancing practices $(n=34,40 \%)$. (e.g. $[74,77,85,90,93,104,106-108,113,118,130,131,138$, $139,142,143,149,153,157,159,165,174])$. These bundles are mainly linked to positive employee outcomes (e.g., improved job satisfaction, retention, and performance) and to a lesser extent to organizational outcomes. Notable is that two studies $[112,141]$ showed inconclusive relationships with job satisfaction and staff retention.

Eleven studies $(12.9 \%)$ considered bundles which combined motivation-enhancing and empowerment enhancing HRM practices [70, 78, 99, 105, 110, 115, 117, 127, $136,140,163]$. These bundles were largely linked to employee outcomes (e.g., improved job satisfaction, motivation) and to a lesser extent to organizational outcomes. Notable, one study reported how a bundle which combined empowerment-enhancing (team work) and motivation-enhancing HRM interventions (flexi-time system, scheduling) failed to reduce staff turnover [78]. 
Table 5 Overview of single HRM practices in relation to performance outcomes

\begin{tabular}{|c|c|c|c|c|c|c|}
\hline Authors, year, country & HRM practices & $\begin{array}{l}\text { Employee } \\
\text { outcome }\end{array}$ & Team outcome & $\begin{array}{l}\text { Organizational } \\
\text { outcome }\end{array}$ & $\begin{array}{l}\text { Patient } \\
\text { outcome }\end{array}$ & $\begin{array}{l}\text { MMAT } \\
\text { Score }\end{array}$ \\
\hline \multicolumn{7}{|l|}{ 1. Training and education } \\
\hline $\begin{array}{l}\text { Ajayi, 2013, Nigeria } \\
\text { [75] }\end{array}$ & training nurses on computer-skills & & & improved efficiency & - & $* * *$ \\
\hline $\begin{array}{l}\text { Eygelaar \& } \\
\text { Stellenberg, 2012, } \\
\text { S.Africa [94] }\end{array}$ & training on nursing care & & & $\begin{array}{l}\text { improved quality } \\
\text { of care }\end{array}$ & - & $* * * *$ \\
\hline $\begin{array}{l}\text { Issahaku et al., } \\
\text { 2012, Ghana [100] }\end{array}$ & $\begin{array}{l}\text { training (clinical and administrative } \\
\text { staff) }\end{array}$ & $\begin{array}{l}\text { improved } \\
\text { performance }\end{array}$ & & & - & $* * * *$ \\
\hline $\begin{array}{l}\text { Jacobs \& Roodt, } \\
\text { 2008, S.Africa [102] }\end{array}$ & $\begin{array}{l}\text { knowledge sharing organizational } \\
\text { culture /learning practice among } \\
\text { professional nurses }\end{array}$ & $\begin{array}{l}\text { reduced turnover } \\
\text { intention }\end{array}$ & & & - & $* * * *$ \\
\hline $\begin{array}{l}\text { Esan et al., 2014, } \\
\text { Nigeria [148] }\end{array}$ & training residence doctors & $\begin{array}{l}\text { improved job } \\
\text { satisfaction }\end{array}$ & & & - & $* * *$ \\
\hline $\begin{array}{l}\text { Letlape et al., 2014, } \\
\text { S.Africa [150] }\end{array}$ & $\begin{array}{l}\text { in-service training on confidence } \\
\text { building }\end{array}$ & & & $\begin{array}{l}\text { improved quality } \\
\text { of care }\end{array}$ & - & ** \\
\hline $\begin{array}{l}\text { Mduma et al., 2015, } \\
\text { Tanzania [155] }\end{array}$ & $\begin{array}{l}\text { simulation training on delivery and } \\
\text { neonatal care }\end{array}$ & & & & $\begin{array}{l}\text { decreased } \\
\text { mortality }\end{array}$ & ** \\
\hline $\begin{array}{l}\text { Bergman et al., } \\
\text { 2008, Tanzania } \\
\text { [146] }\end{array}$ & $\begin{array}{l}\text { trauma team training of physicians } \\
\text { and nurses }\end{array}$ & $\begin{array}{l}\text { improved job } \\
\text { satisfaction }\end{array}$ & $\begin{array}{l}\text { improved team } \\
\text { performance }\end{array}$ & & - & * \\
\hline $\begin{array}{l}\text { Uys et al., 2005, } \\
\text { S.Africa [166] }\end{array}$ & training on supportive supervision & $\begin{array}{l}\text { improved job } \\
\text { satisfaction }\end{array}$ & & $\begin{array}{l}\text { improved quality } \\
\text { of care }\end{array}$ & - & ** \\
\hline $\begin{array}{l}\text { Crofts et al., 2015, } \\
\text { Zimbabwe [172] }\end{array}$ & $\begin{array}{l}\text { onsite-team training on obstetric } \\
\text { emergency care }\end{array}$ & & $\begin{array}{l}\text { improved team } \\
\text { performance in } \\
\text { clinical practices }\end{array}$ & & $\begin{array}{l}\text { improved } \\
\text { maternal } \\
\text { deaths }\end{array}$ & ** \\
\hline \multicolumn{7}{|c|}{ 2. Salary and compensation } \\
\hline $\begin{array}{l}\text { Aberese-Ako et al., } \\
\text { 2014, Ghana [69] }\end{array}$ & $\begin{array}{l}\text { incentives /monthly transport } \\
\text { allowances }\end{array}$ & $\begin{array}{l}\text { improved } \\
\text { performance }\end{array}$ & & & - & $* * * *$ \\
\hline $\begin{array}{l}\text { Nwude \& Uduji, } \\
\text { 2013, Nigeria [120] }\end{array}$ & fair and adequate compensation & $\begin{array}{l}\text { improved job } \\
\text { performance }\end{array}$ & & & - & ** \\
\hline $\begin{array}{l}\text { Atambo et al., 2013, } \\
\text { Kenya [83] }\end{array}$ & implementing incentive systems & $\begin{array}{l}\text { improved } \\
\text { performance }\end{array}$ & & $\begin{array}{l}\text { improved efficiency } \\
\text { of service delivery }\end{array}$ & - & $* * *$ \\
\hline $\begin{array}{l}\text { Ashmore \& Gilson, } \\
\text { 2015, S. Africa [80] }\end{array}$ & $\begin{array}{l}\text { additional wage incentives for } \\
\text { specialists }\end{array}$ & $\begin{array}{l}\text { improved } \\
\text { retention }\end{array}$ & & & - & $* * * *$ \\
\hline \multicolumn{7}{|c|}{ 3. Rostering and scheduling } \\
\hline $\begin{array}{l}\text { Mclntosh \& } \\
\text { Stellenberg, 2009, S. } \\
\text { Africa [154] }\end{array}$ & $\begin{array}{l}\text { implementing staff control strategy/ } \\
\text { scheduling/ to control moonlighting }\end{array}$ & $\begin{array}{l}\text { turnover intention } \\
\text { continued } \\
\text { (not improved) }\end{array}$ & & $\begin{array}{l}\text { improved quality } \\
\text { of care }\end{array}$ & - & ** \\
\hline $\begin{array}{l}\text { Nyathi \& Jooste, } \\
\text { 2008, S. Africa [121] }\end{array}$ & managing reutilization and workload & $\begin{array}{l}\text { reduced } \\
\text { absenteeism } \\
\text { among nurses }\end{array}$ & & & - & $* * *$ \\
\hline $\begin{array}{l}\text { Osisioma et al., } \\
\text { 2015, Nigeria [122] }\end{array}$ & $\begin{array}{l}\text { implementation of flexible working } \\
\text { arrangements }\end{array}$ & $\begin{array}{l}\text { improved } \\
\text { performance }\end{array}$ & & & - & ** \\
\hline $\begin{array}{l}\text { Rispel et al., 2014, } \\
\text { S.Africa [126] }\end{array}$ & $\begin{array}{l}\text { managing rostering \& scheduling to } \\
\text { control moonlighting }\end{array}$ & $\begin{array}{l}\text { reduced intention } \\
\text { to leave }\end{array}$ & & & - & **** \\
\hline \multicolumn{7}{|l|}{ 4. Task shifting } \\
\hline $\begin{array}{l}\text { Ferrinho et al., } \\
\text { 2015, Mozambique } \\
\text { \& Zambia [95] }\end{array}$ & task shifting practice & & & $\begin{array}{l}\text { reduced staff } \\
\text { shortage and } \\
\text { improved quality } \\
\text { of care }\end{array}$ & - & $* * *$ \\
\hline $\begin{array}{l}\text { Jennings et al., } \\
\text { 2011, Benin [103] }\end{array}$ & $\begin{array}{l}\text { task shifting practices for lay nurse } \\
\text { aides }\end{array}$ & & & $\begin{array}{l}\text { improved efficiency } \\
\text { of health care }\end{array}$ & - & $* *$ \\
\hline $\begin{array}{l}\text { Olson et al., 2014, } \\
\text { Malawi [161] }\end{array}$ & $\begin{array}{l}\text { task shifting in patient triage and } \\
\text { treatment }\end{array}$ & & & $\begin{array}{l}\text { improved quality } \\
\text { of care }\end{array}$ & $\begin{array}{l}\text { reduced } \\
\text { inpatient }\end{array}$ & $* * *$ \\
\hline
\end{tabular}


Table 5 Overview of single HRM practices in relation to performance outcomes (Continued)

\begin{tabular}{|c|c|c|c|c|c|c|}
\hline Authors, year, country & HRM practices & $\begin{array}{l}\text { Employee } \\
\text { outcome }\end{array}$ & Team outcome & $\begin{array}{l}\text { Organizational } \\
\text { outcome }\end{array}$ & $\begin{array}{l}\text { Patient } \\
\text { outcome }\end{array}$ & $\begin{array}{l}\text { MMAT } \\
\text { Score }\end{array}$ \\
\hline & & & & & mortality & \\
\hline $\begin{array}{l}\text { Sanjana et al., 2009, } \\
\text { Zamia [164] }\end{array}$ & task shifting for lay counselors & & & $\begin{array}{l}\text { reduced staff shortage, } \\
\text { reduced rate of } \\
\text { errors and }\end{array}$ & - & ** \\
\hline $\begin{array}{l}\text { Galukande et al., } \\
\text { 2013, Uganda [96] }\end{array}$ & task shifting (surgical) practice & & & $\begin{array}{l}\text { improved staff } \\
\text { shortage }\end{array}$ & $\begin{array}{l}\text { decreased } \\
\text { mortality }\end{array}$ & **** \\
\hline $\begin{array}{l}\text { O'Malley et al., } \\
\text { 2014, Namibia [162] }\end{array}$ & task shifting from doctors to nurses & & & $\begin{array}{l}\text { improved quality of } \\
\text { service }\end{array}$ & - & $* * *$ \\
\hline \multicolumn{7}{|l|}{ 5. Managing employees } \\
\hline $\begin{array}{l}\text { Nigussie \& } \\
\text { Demissie, 2013, } \\
\text { Ethiopia [158] }\end{array}$ & leadership styles of nurse managers & $\begin{array}{l}\text { increased job } \\
\text { satisfaction }\end{array}$ & & & - & **** \\
\hline $\begin{array}{l}\text { Okurame, 2009, } \\
\text { Nigeria [160] }\end{array}$ & mentoring practices & $\begin{array}{l}\text { improved job } \\
\text { satisfaction }\end{array}$ & & & - & **** \\
\hline
\end{tabular}

Twenty-one studies (24.7\%) utilized practices from each of the three categories empowerment-, motivation-, and skills-enhancing HRM practices. The results in these studies again mostly present improved employee outcomes (e.g., task performance, retention, motivation, and satisfaction) and some present improved organizational outcomes (e.g., quality of care and efficiency).

The majority of the studies included extrinsic motivation practices, such as salary $(n=40,47.1 \%)$ and supplementary allowances/incentives $(n=27,31.8 \%)$. These financially oriented incentives were most frequently combined with the skills enhancement intervention training (32 studies), and less with empowerment interventions (13 studies). Six studies reported a combination of financial incentives with teamwork [72, 99, 101, 105, 129, 135], and six studies with supervision $[71,114,119,127,129,144]$. In general, these studies reported significant and positive effects on the employee outcomes job satisfaction (13 studies), employee retention (8 studies), and employee performance (9 studies). Two studies, however [78, 111], reported non-significant effects on employee retention, and one study reports a negative effect on job satisfaction [141]. Only three of these financial incentive-related studies reported on organizational performance (i.e., quality of care) $[86,115,129]$.

Scheduling and rostering were also frequently reported motivation-enhancing practices $(n=31$, $36.5 \%)$. Scheduling and rostering were often combined with skills-enhancement interventions (18 studies) and empowerment-enhancing practices (13 studies). Of these studies, 23 reported positive effects on the employee outcomes turnover intention, job satisfaction, and/or employee performance. Positive effects on the organizational outcomes quality of care and reduced waiting time were reported by eight studies.

Leadership/management support practices $(n=24$, $28.2 \%)$ were researched mostly in combination with the skills-enhancing interventions training and staffing, along with the empowerment-enhancing practices team work and supervision. In general, these studies reported significant improvement and positive effects on employee outcomes (e.g., staff retention, job satisfaction and task performance), organizational (e.g., quality of care), and patient outcomes. Some studies $[112,116,171]$ showed inconclusive results on the relationships with employee outcomes and patient satisfaction.

Less frequently researched were bundles using motivation-enhancing practices based on recognition $(n=16,18.8 \%)$ and staff performance appraisal $(n=12,14.1 \%)$, which have often been combined with skills-enhancing training and empowerment-enhancing practices (e.g., task shifting, communication, team work, employee engagement). These studies reported significant improvements and positive effects on employee outcomes (e.g., performance, retention, job satisfaction and intrinsic motivation) and organizational outcomes (e.g., quality of care, reduced waiting time).

\section{Skills-enhancing HRM practices}

Skills-enhancing HRM practices were researched in 66 studies (77.6\%). These studies mostly focused on training, staffing, and mentorship. Only four studies (4.7\%) researched bundles that solely contained skills-enhancing practices [89, 128, 170, 173]. Three of these studies showed significant improvements in organizational outcomes (e.g., efficacy and quality of care) $[89,128,170]$ while one study reported enhanced employee performance [173]. As a side effect, some studies mentioned that trained employees may subsequently leave for better jobs and hence increase turnover. 
Table 6 Overview of HRM bundles in relation to performance outcomes

\begin{tabular}{|c|c|c|c|c|c|c|c|}
\hline \multirow{2}{*}{$\begin{array}{l}\text { Author, year, } \\
\text { country }\end{array}$} & \multicolumn{3}{|l|}{ HRM themes } & \multicolumn{3}{|l|}{ Performance outcomes } & \multirow{2}{*}{$\begin{array}{l}\text { MMAT } \\
\text { Score }\end{array}$} \\
\hline & $\begin{array}{l}\text { Empowerment } \\
\text {-Enhancing } \\
\text { practices }\end{array}$ & $\begin{array}{l}\text { Motivation-Enhancing } \\
\text { practices }\end{array}$ & $\begin{array}{l}\text { Skills- } \\
\text { Enhancing } \\
\text { practices }\end{array}$ & Employee outcome & $\begin{array}{l}\text { Organizational } \\
\text { outcome }\end{array}$ & $\begin{array}{l}\text { Patient } \\
\text { Outcome }\end{array}$ & \\
\hline $\begin{array}{l}\text { Ajemigbitse et al., } \\
\text { 2013, Nigeria [68] }\end{array}$ & $\begin{array}{l}\text { supportive } \\
\text { supervision }\end{array}$ & & $\begin{array}{l}\text { job-based } \\
\text { skill training }\end{array}$ & & $\begin{array}{l}\text { improved } \\
\text { prescribing errors } \\
\text { among junior } \\
\text { physicians }\end{array}$ & & $* * *$ \\
\hline $\begin{array}{l}\text { Ackerman \& Phil, } \\
\text { 2007, S.Africa [70] }\end{array}$ & teamwork & $\begin{array}{l}\text { management support, } \\
\text { scheduling }\end{array}$ & & $\begin{array}{l}\text { improved job } \\
\text { satisfaction }\end{array}$ & & & $* *$ \\
\hline $\begin{array}{l}\text { Francis \& Roger, } \\
\text { 2012, Ghana [71] }\end{array}$ & supervision & $\begin{array}{l}\text { salary, supplementary } \\
\text { allowances, leadership } \\
\text { support, recognition }\end{array}$ & $\begin{array}{l}\text { job-based } \\
\text { skill training }\end{array}$ & $\begin{array}{l}\text { improved retention and } \\
\text { staff motivation }\end{array}$ & & & $* * * *$ \\
\hline $\begin{array}{l}\text { Simiyu \& } \\
\text { Moronge, 2015, } \\
\text { Kenya [72] }\end{array}$ & $\begin{array}{l}\text { teamwork, work- } \\
\text { life balance, } \\
\text { communication } \\
\text { practice }\end{array}$ & $\begin{array}{l}\text { salary, supplementary } \\
\text { benefits }\end{array}$ & recruitment & improved performance & & & $* * * *$ \\
\hline $\begin{array}{l}\text { Allegrazi et al., } \\
\text { 2010, Mali [73] }\end{array}$ & $\begin{array}{l}\text { feedback on } \\
\text { performance }\end{array}$ & & training & & $\begin{array}{l}\text { improved patient } \\
\text { safety }\end{array}$ & & $* * * *$ \\
\hline $\begin{array}{l}\text { Akinyemi \& } \\
\text { Atilola, 2013, } \\
\text { Nigeria [74] }\end{array}$ & & salaries & training & $\begin{array}{l}\text { improved job } \\
\text { satisfaction }\end{array}$ & & & $* * * *$ \\
\hline $\begin{array}{l}\text { Abubeker et al., } \\
\text { 2014, Nigeria [76] }\end{array}$ & & compensation & training & $\begin{array}{l}\text { reduced turnover } \\
\text { intention }\end{array}$ & & & $* * *$ \\
\hline $\begin{array}{l}\text { Asegid et al., } \\
\text { 2014, Ethiopia [77] }\end{array}$ & & $\begin{array}{l}\text { salary, supplementary } \\
\text { allowances }\end{array}$ & training & $\begin{array}{l}\text { improved job } \\
\text { satisfaction and reduced } \\
\text { intention to leave }\end{array}$ & & & $* * * *$ \\
\hline $\begin{array}{l}\text { Ackerman \& } \\
\text { Bezuidenhout, } \\
\text { 2007, S.Africa [78] }\end{array}$ & teamwork & $\begin{array}{l}\text { scheduling(flexi-time } \\
\text { system) }\end{array}$ & & staff turnover(continued) & & & $* * *$ \\
\hline $\begin{array}{l}\text { Ashmore, 2013, } \\
\text { S.Africa [79] }\end{array}$ & & supplementary allowances & $\begin{array}{l}\text { job-based } \\
\text { skill training }\end{array}$ & $\begin{array}{l}\text { improved job } \\
\text { satisfaction and reduced } \\
\text { moonlighting }\end{array}$ & & & $* * * *$ \\
\hline $\begin{array}{l}\text { Nyakundit et al., } \\
\text { 2012, Kenya [81] }\end{array}$ & & recognition, incentives & training & improved performance & $\begin{array}{l}\text { improved quality } \\
\text { of care }\end{array}$ & & $* * *$ \\
\hline $\begin{array}{l}\text { Atambo et al., } \\
\text { 2013, Kenya [82] }\end{array}$ & & recognition, incentives & training & improved performance & $\begin{array}{l}\text { improved } \\
\text { efficiency in } \\
\text { service delivery }\end{array}$ & & $* * * *$ \\
\hline $\begin{array}{l}\text { Aveling et al., } \\
\text { 2015, Rwanda \& } \\
\text { Ethiopia [84] }\end{array}$ & teamwork & & $\begin{array}{l}\text { training, } \\
\text { staffing }\end{array}$ & & $\begin{array}{l}\text { improved quality } \\
\text { of care and } \\
\text { safety of care }\end{array}$ & & $* * * *$ \\
\hline $\begin{array}{l}\text { Awasses et al., } \\
\text { 2013, Namibia } \\
{[85]}\end{array}$ & & $\begin{array}{l}\text { recognition, staff } \\
\text { performance appraisal, } \\
\text { remuneration, } \\
\text { supplementary financial } \\
\text { allowances }\end{array}$ & $\begin{array}{l}\text { in-service } \\
\text { training }\end{array}$ & $\begin{array}{l}\text { improved performance } \\
\text { of nurses }\end{array}$ & & & $* * *$ \\
\hline $\begin{array}{l}\text { Ayeiko et al., 2011, } \\
\text { Kenya [86] }\end{array}$ & $\begin{array}{l}\text { supervision, } \\
\text { feedback }\end{array}$ & & training & & $\begin{array}{l}\text { improved quality } \\
\text { of care }\end{array}$ & & $* * * *$ \\
\hline $\begin{array}{l}\text { Waju et al.,2011, } \\
\text { Ethiopia [87] }\end{array}$ & & management support & $\begin{array}{l}\text { staffing, } \\
\text { training }\end{array}$ & improved performance & & $\begin{array}{l}\text { improved } \\
\text { patient } \\
\text { satisfaction }\end{array}$ & $* * * *$ \\
\hline $\begin{array}{l}\text { Bhengu, 2000, S. } \\
\text { Africa [88] }\end{array}$ & & $\begin{array}{l}\text { rostering \& scheduling, } \\
\text { salary }\end{array}$ & & $\begin{array}{l}\text { reduced intention to } \\
\text { leave improved } \\
\text { motivation }\end{array}$ & & & $* * * *$ \\
\hline $\begin{array}{l}\text { Bradley et al., } \\
\text { 2008, Ethiopia [89] }\end{array}$ & & & $\begin{array}{l}\text { mentorship, } \\
\text { training }\end{array}$ & & $\begin{array}{l}\text { improvement } \\
\text { in hospital } \\
\text { management } \\
\text { skills (efficiency) }\end{array}$ & & $* * * *$ \\
\hline
\end{tabular}


Table 6 Overview of HRM bundles in relation to performance outcomes (Continued)

\begin{tabular}{|c|c|c|c|c|c|c|c|}
\hline \multirow{2}{*}{$\begin{array}{l}\text { Author, year, } \\
\text { country }\end{array}$} & \multicolumn{3}{|l|}{ HRM themes } & \multicolumn{3}{|l|}{ Performance outcomes } & \multirow{2}{*}{$\begin{array}{l}\text { MMAT } \\
\text { Score }\end{array}$} \\
\hline & $\begin{array}{l}\text { Empowerment } \\
\text {-Enhancing } \\
\text { practices }\end{array}$ & $\begin{array}{l}\text { Motivation-Enhancing } \\
\text { practices }\end{array}$ & $\begin{array}{l}\text { Skills- } \\
\text { Enhancing } \\
\text { practices }\end{array}$ & Employee outcome & $\begin{array}{l}\text { Organizational } \\
\text { outcome }\end{array}$ & $\begin{array}{l}\text { Patient } \\
\text { Outcome }\end{array}$ & \\
\hline $\begin{array}{l}\text { McAuliffe, 2009, } \\
\text { Malawi [90] }\end{array}$ & & & & improved staff retention & & & \\
\hline $\begin{array}{l}\text { Dagne et al., 2015, } \\
\text { Ethiopia [91] }\end{array}$ & $\begin{array}{l}\text { communication/ } \\
\text { supervisor } \\
\text { feedback }\end{array}$ & $\begin{array}{l}\text { job content management } \\
\text { of schedules, } \\
\text { performance review, } \\
\text { financial incentives, } \\
\text { recognition }\end{array}$ & staffing & $\begin{array}{l}\text { improved motivation of } \\
\text { health professionals }\end{array}$ & $\begin{array}{l}\text { improved quality } \\
\text { of care }\end{array}$ & & $* * * *$ \\
\hline $\begin{array}{l}\text { De Brouwere et } \\
\text { al., 2009, Senegal } \\
\text { [92] }\end{array}$ & $\begin{array}{l}\text { teamwork, task } \\
\text { shifting }\end{array}$ & & & & & $\begin{array}{l}\text { improved } \\
\text { maternal } \\
\text { mortality }\end{array}$ & $* * *$ \\
\hline $\begin{array}{l}\text { Dieleman et al., } \\
\text { 2006, Mali [93] }\end{array}$ & & $\begin{array}{l}\text { salary, performance } \\
\text { appraisal, reward } \\
\text { system }\end{array}$ & training & improved motivation & & & $* * * *$ \\
\hline $\begin{array}{l}\text { Hall, 2004, S. } \\
\text { Africa [97] }\end{array}$ & & $\begin{array}{l}\text { salary, supplementary } \\
\text { incentives/ allowances, } \\
\text { scheduling }\end{array}$ & & $\begin{array}{l}\text { reduced intention to } \\
\text { leave }\end{array}$ & & & $* *$ \\
\hline $\begin{array}{l}\text { Honda \& Vio, } \\
\text { 2015, } \\
\text { Mozambique [98] }\end{array}$ & & $\begin{array}{l}\text { incentives, scheduling, } \\
\text { salaries }\end{array}$ & $\begin{array}{l}\text { job-based } \\
\text { skill training }\end{array}$ & $\begin{array}{l}\text { improved job } \\
\text { satisfaction and } \\
\text { retention }\end{array}$ & & & $* * *$ \\
\hline $\begin{array}{l}\text { Libeziako et al., } \\
\text { 2013, S. Africa [99] }\end{array}$ & $\begin{array}{l}\text { teamwork } \\
\text { practice }\end{array}$ & $\begin{array}{l}\text { salary, supplementary } \\
\text { allowances }\end{array}$ & & improved motivation & & & $* *$ \\
\hline $\begin{array}{l}\text { Jack, 2013, Ghana } \\
\text { [101] }\end{array}$ & teamwork & $\begin{array}{l}\text { compensation, } \\
\text { allowances }\end{array}$ & $\begin{array}{l}\text { recruitment/ } \\
\text { staffing, } \\
\text { training }\end{array}$ & improved retention & & & $* *$ \\
\hline $\begin{array}{l}\text { Kamanzi \& Nikosi, } \\
\text { 2011, Rwanda } \\
\text { [104] }\end{array}$ & & $\begin{array}{l}\text { remuneration, } \\
\text { recognition }\end{array}$ & $\begin{array}{l}\text { job-based } \\
\text { skill training }\end{array}$ & $\begin{array}{l}\text { improved level of } \\
\text { motivation }\end{array}$ & & & $* * * *$ \\
\hline $\begin{array}{l}\text { Kekana et al., } \\
\text { 2007, S.Africa } \\
\text { [105] }\end{array}$ & teamwork & $\begin{array}{l}\text { performance appraisal, } \\
\text { remuneration, } \\
\text { scheduling }\end{array}$ & & $\begin{array}{l}\text { improved job } \\
\text { satisfaction }\end{array}$ & & & $* * * *$ \\
\hline $\begin{array}{l}\text { Khamis \& Njau, } \\
\text { 2014, Tanzania } \\
\text { [106] }\end{array}$ & & $\begin{array}{l}\text { salary, allowances, } \\
\text { management support, } \\
\text { rostering \& scheduling }\end{array}$ & $\begin{array}{l}\text { staffing, } \\
\text { training }\end{array}$ & & $\begin{array}{l}\text { improved quality } \\
\text { of care at } \\
\text { outpatient }\end{array}$ & & $* * * *$ \\
\hline $\begin{array}{l}\text { Kotzee \& Couper, } \\
2006, \text { S. Africa } \\
\text { [107] }\end{array}$ & & $\begin{array}{l}\text { salaries, allowances, } \\
\text { recognition }\end{array}$ & $\begin{array}{l}\text { training, } \\
\text { mentorship }\end{array}$ & $\begin{array}{l}\text { improved retention of } \\
\text { doctors }\end{array}$ & & & $* * * *$ \\
\hline $\begin{array}{l}\text { Kruger \& } \\
\text { Bezuidenhout, } \\
\text { 2015, S. Africa } \\
\text { [108] }\end{array}$ & & $\begin{array}{l}\text { scheduling, promotion, } \\
\text { management support }\end{array}$ & training & $\begin{array}{l}\text { reduced female doctors } \\
\text { dissatisfaction in } \\
\text { balancing professional } \\
\text { work and family lives }\end{array}$ & & & $* * * *$ \\
\hline $\begin{array}{l}\text { Liphoko et al., } \\
\text { 2006, S.Africa } \\
\text { [109] }\end{array}$ & & $\begin{array}{l}\text { performance appraisal, } \\
\text { promotion, management } \\
\text { support }\end{array}$ & $\begin{array}{l}\text { job-based } \\
\text { skill training }\end{array}$ & $\begin{array}{l}\text { improved job } \\
\text { satisfaction of nurses }\end{array}$ & & & $* * *$ \\
\hline $\begin{array}{l}\text { Leshabari et al., } \\
\text { 2008, Tanzania } \\
\text { [110] }\end{array}$ & $\begin{array}{l}\text { communication/ } \\
\text { feedback }\end{array}$ & $\begin{array}{l}\text { performance evaluation, } \\
\text { salaries }\end{array}$ & & $\begin{array}{l}\text { improved job } \\
\text { satisfaction and } \\
\text { motivation }\end{array}$ & & & $* * * *$ \\
\hline $\begin{array}{l}\text { Longmore \& } \\
\text { Ronnie, 2014, } \\
\text { S.Africa [111] }\end{array}$ & communication & $\begin{array}{l}\text { salaries, performance } \\
\text { appraisal }\end{array}$ & training & $\begin{array}{l}\text { improved retention of } \\
\text { doctors }\end{array}$ & & & * \\
\hline $\begin{array}{l}\text { Luboga et al., } \\
\text { 2011, Uganda } \\
\text { [112] }\end{array}$ & & $\begin{array}{l}\text { compensation/salaries, } \\
\text { benefits, recognition, } \\
\text { scheduling workload, } \\
\text { management support }\end{array}$ & $\begin{array}{l}\text { training, } \\
\text { staffing }\end{array}$ & $\begin{array}{l}\text { improved job } \\
\text { satisfaction and } \\
\text { retention of physicians }\end{array}$ & & & $* *$ \\
\hline $\begin{array}{l}\text { Makapela \& Useh, } \\
\text { 2015, S.Africa }\end{array}$ & & $\begin{array}{l}\text { salary, management } \\
\text { support, allowance }\end{array}$ & $\begin{array}{l}\text { job-based } \\
\text { skill training }\end{array}$ & improved retention & & & $* * *$ \\
\hline
\end{tabular}


Table 6 Overview of HRM bundles in relation to performance outcomes (Continued)

\begin{tabular}{|c|c|c|c|c|c|c|c|}
\hline \multirow{2}{*}{$\begin{array}{l}\text { Author, year, } \\
\text { country }\end{array}$} & \multicolumn{3}{|l|}{ HRM themes } & \multicolumn{3}{|l|}{ Performance outcomes } & \multirow{2}{*}{$\begin{array}{l}\text { MMAT } \\
\text { Score }\end{array}$} \\
\hline & $\begin{array}{l}\text { Empowerment } \\
\text {-Enhancing } \\
\text { practices }\end{array}$ & $\begin{array}{l}\text { Motivation-Enhancing } \\
\text { practices }\end{array}$ & $\begin{array}{l}\text { Skills- } \\
\text { Enhancing } \\
\text { practices }\end{array}$ & Employee outcome & $\begin{array}{l}\text { Organizational } \\
\text { outcome }\end{array}$ & $\begin{array}{l}\text { Patient } \\
\text { Outcome }\end{array}$ & \\
\hline \multicolumn{8}{|l|}{ [113] } \\
\hline $\begin{array}{l}\text { Mathauer \& } \\
\text { Imhoff, 2006, } \\
\text { Benin \& Kenya } \\
\text { [114] }\end{array}$ & supervision & $\begin{array}{l}\text { recognition, allowances, } \\
\text { salary }\end{array}$ & $\begin{array}{l}\text { job-based } \\
\text { skill training }\end{array}$ & improved motivation & & & $* * * *$ \\
\hline $\begin{array}{l}\text { Mbindyo et al., } \\
\text { 2009, Kenya [115] }\end{array}$ & $\begin{array}{l}\text { employee } \\
\text { engagement, } \\
\text { communication }\end{array}$ & $\begin{array}{l}\text { promotion, leadership } \\
\text { support, performance } \\
\text { appraisal, incentives }\end{array}$ & & & $\begin{array}{l}\text { improved quality } \\
\text { of care }\end{array}$ & & $* * * *$ \\
\hline $\begin{array}{l}\text { McAuliffe et al., } \\
\text { 2009, Malawi } \\
\text { [116] }\end{array}$ & teamwork & management support & staffing & $\begin{array}{l}\text { improved task } \\
\text { performance }\end{array}$ & & & $* *$ \\
\hline $\begin{array}{l}\text { McAuliffe et al., } \\
\text { 2013, Malawi, } \\
\text { Tanzania \& } \\
\text { Mozambique } \\
\text { [117] }\end{array}$ & $\begin{array}{l}\text { job autonomy, } \\
\text { task shifting, } \\
\text { teamwork, } \\
\text { supervision }\end{array}$ & leadership support & & $\begin{array}{l}\text { improved job } \\
\text { satisfaction and reduced } \\
\text { intention to leave }\end{array}$ & & & $* * *$ \\
\hline $\begin{array}{l}\text { Mokoka et al., } \\
\text { 2010, S.Africa } \\
\text { [118] }\end{array}$ & & $\begin{array}{l}\text { salary, rostering \& } \\
\text { scheduling, management } \\
\text { support }\end{array}$ & training & $\begin{array}{l}\text { improved retention of } \\
\text { nurses }\end{array}$ & & & $* * *$ \\
\hline $\begin{array}{l}\text { Mubyazi et al., } \\
\text { 2012, Tanzania } \\
\text { [119] }\end{array}$ & supervision & Incentives & $\begin{array}{l}\text { staffing, } \\
\text { training }\end{array}$ & improved motivation & & & $* * *$ \\
\hline $\begin{array}{l}\text { Pieterson, 2005, } \\
\text { S.Africa [123] }\end{array}$ & & $\begin{array}{l}\text { pay, management } \\
\text { support, scheduling, } \\
\text { promotion }\end{array}$ & & $\begin{array}{l}\text { improved job } \\
\text { satisfaction }\end{array}$ & & & $* * *$ \\
\hline $\begin{array}{l}\text { Pillay, 2009, } \\
\text { S.Africa [124] }\end{array}$ & $\begin{array}{l}\text { teamwork, job } \\
\text { autonomy, job } \\
\text { security }\end{array}$ & rostering \& scheduling & training & $\begin{array}{l}\text { improved job } \\
\text { satisfaction and } \\
\text { motivation }\end{array}$ & & & $* * * *$ \\
\hline $\begin{array}{l}\text { Prytherch et al., } \\
\text { 2012, Tanzania } \\
\text { [125] }\end{array}$ & & $\begin{array}{l}\text { rostering \& scheduling, } \\
\text { salaries, incentives, } \\
\text { recognition/promotion }\end{array}$ & & $\begin{array}{l}\text { increased job } \\
\text { performance }\end{array}$ & & & $* * *$ \\
\hline $\begin{array}{l}\text { Selebi \& Minnaar, } \\
\text { 2007, S.Africa } \\
\text { [127] }\end{array}$ & $\begin{array}{l}\text { supportive } \\
\text { supervision }\end{array}$ & salaries & & $\begin{array}{l}\text { improved job } \\
\text { satisfaction }\end{array}$ & & & $* * *$ \\
\hline $\begin{array}{l}\text { Sikwese et al., } \\
\text { 2010, Zambia } \\
\text { [128] }\end{array}$ & & & $\begin{array}{l}\text { staffing/ } \\
\text { selection, } \\
\text { training }\end{array}$ & & $\begin{array}{l}\text { improved } \\
\text { efficiency of } \\
\text { service delivery }\end{array}$ & & $* * *$ \\
\hline $\begin{array}{l}\text { Siril et al., 2011, } \\
\text { Tanzania [129] }\end{array}$ & $\begin{array}{l}\text { supervision, } \\
\text { teamwork }\end{array}$ & $\begin{array}{l}\text { compensation, rostering \& } \\
\text { scheduling }\end{array}$ & training & & $\begin{array}{l}\text { improved quality } \\
\text { of care }\end{array}$ & & $* * * *$ \\
\hline $\begin{array}{l}\text { Ssengooba et al., } \\
\text { 2002, Uganda } \\
{[130]}\end{array}$ & & rostering \& scheduling & staffing & & $\begin{array}{l}\text { improved hospital } \\
\text { performance } \\
\text { (efficiency and } \\
\text { effectiveness) }\end{array}$ & & $* *$ \\
\hline $\begin{array}{l}\text { Stodel \& Stewart- } \\
\text { Smith, 2011, } \\
\text { S.Africa [131] }\end{array}$ & supervision & scheduling & $\begin{array}{l}\text { training, } \\
\text { mentorship }\end{array}$ & improved retention & & & $* * *$ \\
\hline $\begin{array}{l}\text { Tabatabai et al., } \\
\text { 2013, Tanzania } \\
\text { [132] }\end{array}$ & $\begin{array}{l}\text { employee } \\
\text { engagement }\end{array}$ & $\begin{array}{l}\text { salary, incentives, } \\
\text { scheduling, management } \\
\text { support }\end{array}$ & training & $\begin{array}{l}\text { reduced internal } \\
\text { migration (public to } \\
\text { private) }\end{array}$ & & & $* *$ \\
\hline $\begin{array}{l}\text { Thatte \& Choi, } \\
\text { 2014, Kenya [133] }\end{array}$ & supervision & & $\begin{array}{l}\text { written job } \\
\text { descriptions, } \\
\text { training }\end{array}$ & & $\begin{array}{l}\text { improved service } \\
\text { quality }\end{array}$ & & $* *$ \\
\hline $\begin{array}{l}\text { Uwaliraye et al., } \\
\text { 2013, Rwanda }\end{array}$ & feedback & & training & $\begin{array}{l}\text { improved performance } \\
\text { of nurses and midwives }\end{array}$ & & & $* * *$ \\
\hline
\end{tabular}


Table 6 Overview of HRM bundles in relation to performance outcomes (Continued)

\begin{tabular}{|c|c|c|c|c|c|c|c|}
\hline \multirow{2}{*}{$\begin{array}{l}\text { Author, year, } \\
\text { country }\end{array}$} & \multicolumn{3}{|l|}{ HRM themes } & \multicolumn{3}{|l|}{ Performance outcomes } & \multirow{2}{*}{$\begin{array}{l}\text { MMAT } \\
\text { Score }\end{array}$} \\
\hline & $\begin{array}{l}\text { Empowerment } \\
\text {-Enhancing } \\
\text { practices }\end{array}$ & $\begin{array}{l}\text { Motivation-Enhancing } \\
\text { practices }\end{array}$ & $\begin{array}{l}\text { Skills- } \\
\text { Enhancing } \\
\text { practices }\end{array}$ & Employee outcome & $\begin{array}{l}\text { Organizational } \\
\text { outcome }\end{array}$ & $\begin{array}{l}\text { Patient } \\
\text { Outcome }\end{array}$ & \\
\hline $\begin{array}{l}\text { Yami et al., 2011, } \\
\text { Ethiopia [135] }\end{array}$ & teamwork & $\begin{array}{l}\text { supplementary } \\
\text { allowances, salary }\end{array}$ & training & $\begin{array}{l}\text { improved job } \\
\text { satisfaction }\end{array}$ & & & $* * * *$ \\
\hline $\begin{array}{l}\text { Bekker et al., 2015, } \\
\text { S. Africa [136] }\end{array}$ & communication & rostering \& scheduling & & $\begin{array}{l}\text { enhanced job } \\
\text { satisfaction }\end{array}$ & & & $* * *$ \\
\hline $\begin{array}{l}\text { Chandler et al., } \\
\text { 2009, Tanzania } \\
\text { [137] }\end{array}$ & & $\begin{array}{l}\text { salary, management } \\
\text { support, rostering \& } \\
\text { scheduling }\end{array}$ & training & improved performance & $\begin{array}{l}\text { improved quality } \\
\text { of care }\end{array}$ & & $* * * *$ \\
\hline $\begin{array}{l}\text { Chi et al., 2015, } \\
\text { Burundi \& Uganda } \\
\text { [138] }\end{array}$ & & $\begin{array}{l}\text { rostering \& scheduling, } \\
\text { remuneration }\end{array}$ & staffing & & $\begin{array}{l}\text { improved quality } \\
\text { of maternal care }\end{array}$ & & $* * *$ \\
\hline $\begin{array}{l}\text { Chirwa, 2000, } \\
\text { Malawi [139] }\end{array}$ & & performance appraisal & Staffing & & $\begin{array}{l}\text { improved quality } \\
\text { of care }\end{array}$ & & $* * *$ \\
\hline $\begin{array}{l}\text { Hollup, 2012, } \\
\text { Mauritius [140] }\end{array}$ & $\begin{array}{l}\text { job security and } \\
\text { safety }\end{array}$ & salary & & $\begin{array}{l}\text { improved staff } \\
\text { motivation }\end{array}$ & & & $* * * *$ \\
\hline $\begin{array}{l}\text { Klopper et al., } \\
\text { 2012, S.Africa } \\
\text { [141] }\end{array}$ & & $\begin{array}{l}\text { wages, study leave } \\
\text { opportunities }\end{array}$ & $\begin{array}{l}\text { skills-training } \\
\text { for career } \\
\text { advancement }\end{array}$ & job dissatisfaction & & & $* *$ \\
\hline $\begin{array}{l}\text { Lasebitan \& } \\
\text { Oyetundt, 2012, } \\
\text { Nigeria [142] }\end{array}$ & & $\begin{array}{l}\text { rostering \& scheduling, } \\
\text { wages }\end{array}$ & staffing & improved retention & & & $* * * *$ \\
\hline $\begin{array}{l}\text { Mudaly \& Nkosi, } \\
\text { 2015, S.Africa } \\
\text { [143] }\end{array}$ & & $\begin{array}{l}\text { scheduling, promotion, } \\
\text { pay, rewards/incentives }\end{array}$ & $\begin{array}{l}\text { training, } \\
\text { staffing }\end{array}$ & reduced absenteeism & & & $* * *$ \\
\hline $\begin{array}{l}\text { Tibandebage et } \\
\text { al., 2015, Tanzania } \\
\text { [144] }\end{array}$ & supervision & $\begin{array}{l}\text { incentives, salaries, } \\
\text { leadership support, } \\
\text { rostering \& scheduling }\end{array}$ & Staffing & improved performance & & & $* * *$ \\
\hline $\begin{array}{l}\text { Courtright et al., } \\
\text { 2007, Malawi, } \\
\text { Uganda, Tanzania } \\
\text { \& Kenya [145] }\end{array}$ & supervision & management support & Training & improved performance & & & $* * * *$ \\
\hline $\begin{array}{l}\text { Doherty et al., } \\
\text { 2013, S.Africa } \\
\text { [147] }\end{array}$ & $\begin{array}{l}\text { supervision, task } \\
\text { shifting }\end{array}$ & & & & $\begin{array}{l}\text { improved quality } \\
\text { of care, reduced } \\
\text { staff shortage and } \\
\text { workload }\end{array}$ & & $* * *$ \\
\hline $\begin{array}{l}\text { Kamau \& Omondi, } \\
\text { 2015, Kenya [149] }\end{array}$ & & $\begin{array}{l}\text { supplementary } \\
\text { allowances/incentives }\end{array}$ & $\begin{array}{l}\text { job-based } \\
\text { skill training }\end{array}$ & improved staff retention & & & $* * * *$ \\
\hline $\begin{array}{l}\text { Madzimbamuto et } \\
\text { al., 2014, } \\
\text { Botswana [151] }\end{array}$ & supervision & & Training & & $\begin{array}{l}\text { improved quality } \\
\text { of care }\end{array}$ & & $* *$ \\
\hline $\begin{array}{l}\text { Mahlo \& Muller, } \\
\text { 2000, S.Africa } \\
\text { [152] }\end{array}$ & communication & & Training & & $\begin{array}{l}\text { improved quality } \\
\text { of care }\end{array}$ & & $* * * *$ \\
\hline $\begin{array}{l}\text { Manongi et al., } \\
\text { 2009, Tanzania } \\
\text { [153] }\end{array}$ & & Salary & Training & improved performance & & & $* * *$ \\
\hline $\begin{array}{l}\text { Nabirye, 2010, } \\
\text { Uganda [156] }\end{array}$ & & $\begin{array}{l}\text { scheduling, pay, } \\
\text { incentives/allowances }\end{array}$ & & $\begin{array}{l}\text { improved performance } \\
\text { of nurses and job } \\
\text { satisfaction }\end{array}$ & & & $* * *$ \\
\hline $\begin{array}{l}\text { Ndetei et al., 2008, } \\
\text { Kenya [157] }\end{array}$ & & Salary & Training & $\begin{array}{l}\text { reduced migration of } \\
\text { health workforce } \\
\text { (retention) }\end{array}$ & & & $* * *$ \\
\hline $\begin{array}{l}\text { Okeke, 2008, } \\
\text { Nigeria [159] }\end{array}$ & & salary & Recruitment & improved retention & & & $* *$ \\
\hline Rauf et al., 2008, & task shifting & scheduling, performance & & & reduced waiting & & $* *$ \\
\hline
\end{tabular}


Table 6 Overview of HRM bundles in relation to performance outcomes (Continued)

\begin{tabular}{|c|c|c|c|c|c|c|c|}
\hline \multirow{2}{*}{$\begin{array}{l}\text { Author, year, } \\
\text { country }\end{array}$} & \multicolumn{3}{|l|}{ HRM themes } & \multicolumn{3}{|l|}{ Performance outcomes } & \multirow{2}{*}{$\begin{array}{l}\text { MMAT } \\
\text { Score }\end{array}$} \\
\hline & $\begin{array}{l}\text { Empowerment } \\
\text {-Enhancing } \\
\text { practices }\end{array}$ & $\begin{array}{l}\text { Motivation-Enhancing } \\
\text { practices }\end{array}$ & $\begin{array}{l}\text { Skills- } \\
\text { Enhancing } \\
\text { practices }\end{array}$ & Employee outcome & $\begin{array}{l}\text { Organizational } \\
\text { outcome }\end{array}$ & $\begin{array}{l}\text { Patient } \\
\text { Outcome }\end{array}$ & \\
\hline S.Africa [163] & & evaluation/appraisal & & & $\begin{array}{l}\text { time (maximized } \\
\text { efficiency) }\end{array}$ & & \\
\hline $\begin{array}{l}\text { Thomas \& Valli, } \\
\text { 2006, S.Africa } \\
\text { [165] }\end{array}$ & & scheduling, salary & $\begin{array}{l}\text { training, } \\
\text { staffing }\end{array}$ & $\begin{array}{l}\text { improved job } \\
\text { satisfaction }\end{array}$ & & & $* * * *$ \\
\hline $\begin{array}{l}\text { Yeboha et al., } \\
\text { 2014, Ghana [167] }\end{array}$ & communication & management support & Training & improved retention & & & $* * *$ \\
\hline $\begin{array}{l}\text { Rawlins et al., } \\
\text { 2003, Kenya [168] }\end{array}$ & $\begin{array}{l}\text { feedback, } \\
\text { teamwork }\end{array}$ & management support & $\begin{array}{l}\text { staffing, } \\
\text { written job } \\
\text { descriptions }\end{array}$ & & $\begin{array}{l}\text { improved } \\
\text { organizational } \\
\text { performance } \\
\text { (efficiency) }\end{array}$ & & $* * *$ \\
\hline $\begin{array}{l}\text { Giuseppe et al., } \\
\text { 2002, Kenya [169] }\end{array}$ & $\begin{array}{l}\text { communication, } \\
\text { work-life } \\
\text { balance }\end{array}$ & $\begin{array}{l}\text { scheduling, management } \\
\text { support }\end{array}$ & Training & $\begin{array}{l}\text { improved task } \\
\text { performance and } \\
\text { improved retention of } \\
\text { resident doctors }\end{array}$ & & & $* * *$ \\
\hline $\begin{array}{l}\text { Ngao, 2013, Kenya } \\
\text { [170] }\end{array}$ & & & $\begin{array}{l}\text { recruitment/ } \\
\text { staffing, } \\
\text { training, } \\
\text { mentorship }\end{array}$ & & $\begin{array}{l}\text { improved quality } \\
\text { of care }\end{array}$ & & $* *$ \\
\hline $\begin{array}{l}\text { Kotagal et al., } \\
\text { 2009, Rwanda } \\
\text { [171] }\end{array}$ & & leadership support & staffing & & & $\begin{array}{l}\text { improved } \\
\text { patient } \\
\text { satisfaction }\end{array}$ & $* *$ \\
\hline $\begin{array}{l}\text { Dowing, 2016, } \\
\text { Uganda [173] }\end{array}$ & & & $\begin{array}{l}\text { training, } \\
\text { mentorship }\end{array}$ & $\begin{array}{l}\text { improved nurses' } \\
\text { performance }\end{array}$ & & & $* * *$ \\
\hline $\begin{array}{l}\text { Faye et al., 2013, } \\
\text { Senegal \& Mali } \\
{[174]}\end{array}$ & & $\begin{array}{l}\text { salary, supplementary } \\
\text { allowances, scheduling, } \\
\text { management support }\end{array}$ & training & $\begin{array}{l}\text { improved job } \\
\text { satisfaction }\end{array}$ & & & $* * * *$ \\
\hline $\begin{array}{l}\text { Doef et al., 2011, } \\
\text { Kenya, Tanzania \& } \\
\text { Uganda [175] }\end{array}$ & & $\begin{array}{l}\text { scheduling, management } \\
\text { support, supplementary } \\
\text { allowances }\end{array}$ & staffing & $\begin{array}{l}\text { improved job } \\
\text { satisfaction and reduced } \\
\text { level of burnout }\end{array}$ & & & $* * * *$ \\
\hline $\begin{array}{l}\text { Srofenyoh et al., } \\
\text { 2012, Ghana [176] }\end{array}$ & $\begin{array}{l}\text { teamwork, } \\
\text { communication }\end{array}$ & leadership support & training & $\begin{array}{l}\text { improved employee } \\
\text { performance }\end{array}$ & & $\begin{array}{l}\text { improved } \\
\text { patient } \\
\text { satisfaction } \\
\text { and clinical } \\
\text { outcomes }\end{array}$ & $* * *$ \\
\hline $\begin{array}{l}\text { Woldegabriel et } \\
\text { al., 2016, Ethiopia } \\
\text { [177] }\end{array}$ & communication & $\begin{array}{l}\text { scheduling, performance } \\
\text { appraisal }\end{array}$ & $\begin{array}{l}\text { selection/ } \\
\text { recruitment, } \\
\text { training }\end{array}$ & $\begin{array}{l}\text { improved intrinsic } \\
\text { motivation of health } \\
\text { workforce }\end{array}$ & & & **** \\
\hline $\begin{array}{l}\text { Puoane et al., } \\
\text { 2008, S.Africa } \\
\text { [178] }\end{array}$ & $\begin{array}{l}\text { teamwork, } \\
\text { supervision, } \\
\text { feedback }\end{array}$ & $\begin{array}{l}\text { leadership support, } \\
\text { monitoring performance }\end{array}$ & $\begin{array}{l}\text { in-service } \\
\text { training and } \\
\text { induction of } \\
\text { new nurses }\end{array}$ & $\begin{array}{l}\text { improved task } \\
\text { performance }\end{array}$ & $\begin{array}{l}\text { improved quality } \\
\text { of care in the } \\
\text { better performing } \\
\text { hospitals }\end{array}$ & & $* * * *$ \\
\hline
\end{tabular}

As mentioned above, 34 studies report on bundles combining skills-enhancing practices with motivationenhancing practices. Eight studies combined skills-enhancing practices (e.g., training, staffing) with empowerment-enhancing practices (e.g., supervision, feedback, teamwork) $[68,73,84,86,133,134,151,152]$. They mostly reported significant positive effects on organizational outcomes (e.g., quality of care and patient safety).

Among the skills-enhancing practices, training occurred most frequently (50 studies), followed by staffing and recruitment practices (23 studies). Most of these studies were associated with employee outcomes (e.g., retention, task performance, job satisfaction, and motivation), and less with organizational outcome (e.g., quality of care) (13 studies) and patient outcomes (2 studies). Only one study researched skills-enhancing training combined with motivation-enhancing practice (i.e., supplementary allowances) and showed improved employee outcomes (i.e., job satisfaction and reduced moonlighting) [79]. Two studies showed that written job descriptions (in combination with training, staffing, and empowerment- and motivation-enhancing practices) 
yielded significantly positive effects on organizational outcomes (i.e., efficiency and quality of care) [133, 168].

\section{Empowerment-enhancing HRM practices}

Empowerment-enhancing practices $(n=42,49.4 \%)$ mainly entailed teamwork, communication, and supportive supervision. Only two studies considered purely empowerment-enhancing bundles, one of which showed improvements in the patient outcome maternal mortality [92], and the other reported improvement in the organizational outcomes quality of care and staff shortage [147].

Most studies $(n=30,35.3 \%)$ that addressed empower ment-enhancing practices considered one empowermentrelated practice combined with other practices. Eleven studies researched empowerment-enhancing practices (e.g., team work, supervision) combined with motivation-enhancing practices (e.g., compensation, scheduling) [70, 78, 99, $105,110,115,117,127,136,140,163]$. These studies mostly reported improvement on employee outcomes (e.g., satisfaction, retention, performance). Some reported improvement on organizational outcomes (e.g., quality of care, efficiency) and patient experience (i.e., satisfaction and clinical outcomes). However, one study [78] reported no improvement on the employee outcome turnover intention.

Communication/feedback practices (16 studies), teamwork (15 studies), and supervision (14 studies) occurred most frequently in combination with skills- and motivation-enhancing practices. Most of these studies were associated with improved employee satisfaction, motivation, retention, and performance. Nine studies reported improvement on the organizational outcomes (e.g., quality of care) and to a lesser extent to patient outcome [68, 73, 84, 86, 87, 91, 129, 133, 168].

Of the empowerment-enhancing practices, employee engagement, work-life balance, job autonomy, job security, and safety were less frequently researched (six studies). Of these studies, six reported positive effects and improvement on employee outcomes (e.g., job satisfaction, motivation, retention, task performance) [72, 117, 124, 132, 140, 169]. Positive significant effects on the organizational outcome quality of care were reported once [115].

\section{Conclusion}

For the first time, an overview of studies that researched the link between HRM and performance in SSA hospitals is presented. The literature shows that HRM affects four different categories of performance outcomes: (individual) employee, team, organization (as a whole), and patient outcomes. Employee outcomes and organizational outcomes are frequently researched, whereas team outcomes and patient outcomes are significantly less researched. Evidence of the effect of HRM on patient outcomes, probably mediated via HRM outcomes, for now primarily builds on studies outside the SSA and studies with low quality of evidence within the SSA setting [17, 19, 24, 39]. Given the scarcity of human resources and the disproportional high burden of disease in SSA, further research on the effect of HRM practices on patient outcomes in SSA contexts is urgently called for. As previous studies reveal that contextual characteristics impact outcomes [16, 18, 33], contextual characteristics need to be taken into account, as can be attained by adopting the Context, Intervention, Mechanism, Outcome (CIMO) logic [52, 179, 180].

This review revealed 18 types of HRM practices that were researched in relation to performance of SSA hospitals. As shown in Table 7, this number is comparable to the 26 types of HRM practices presented by Boselie et al. [19]; 13 HRM practices (within high-performance work practice) shown by Combs et al. [20]; 10 HRM practices acknowledged by Hyde et al. [26]; and 6 HRM practices presented by Dieleman et al. [16]. Table 7 summarizes several reviews on HRM in different settings and shows that there is overlap in HRM practices. For example, training and education, compensation, recruitment, and team working are shown to be effective in many reviews. Although there is overlap in HRM practices researched in SSA context and the above mentions studies that researched HRM practices in a broader context (e.g., training, pay, and reward), three areas are under-explored in SSA. First, in SSA context, HRM practices related to employment are only researched in terms of staffing, rostering, and scheduling, but not in terms of selection, diversity, equal opportunity, exit management, and egalitarianism. On the other hand, employment regarding moonlighting is explored in SSA context, but rarely in overall HRM literature. Second, direct participation is studied in terms of communication, empowerment, and management, but not in terms of indirect participations through committees and councils, or in terms of socialization and social responsibility practices. Third, the professionalization of HRM function/department as a HRM practice is not researched at all in SSA context. These differences could be explained by the difficult SSA labor market that is characterized with low wages, the collectivistic and hierarchical organizational culture, and the lack of officially appointed HR functions.

The minority of included studies focused on single HRM practices. They mostly found positive effects on performance. Most included studies reported on implementation of HRM bundles, as is in line with Subramony [28] and Boselie et al. who claim that HRM bundles are likely to be synergistic, thus yielding stronger effects on performance than single HRM practices [19].

\section{Single HRM practices versus HRM bundles}

In SSA, training and education are the most researched single HRM practice. Training is one of seven Pfeffer's best 


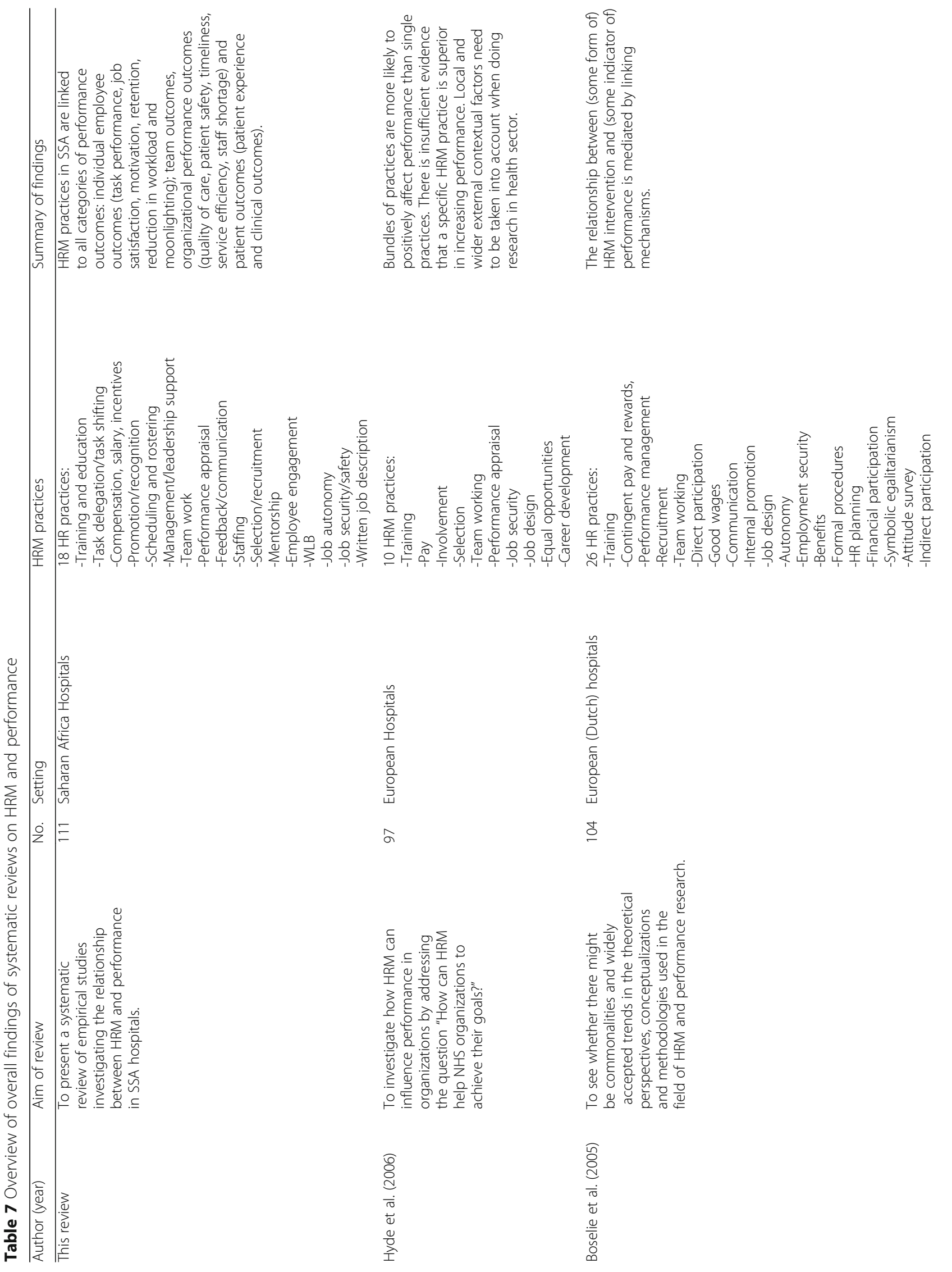




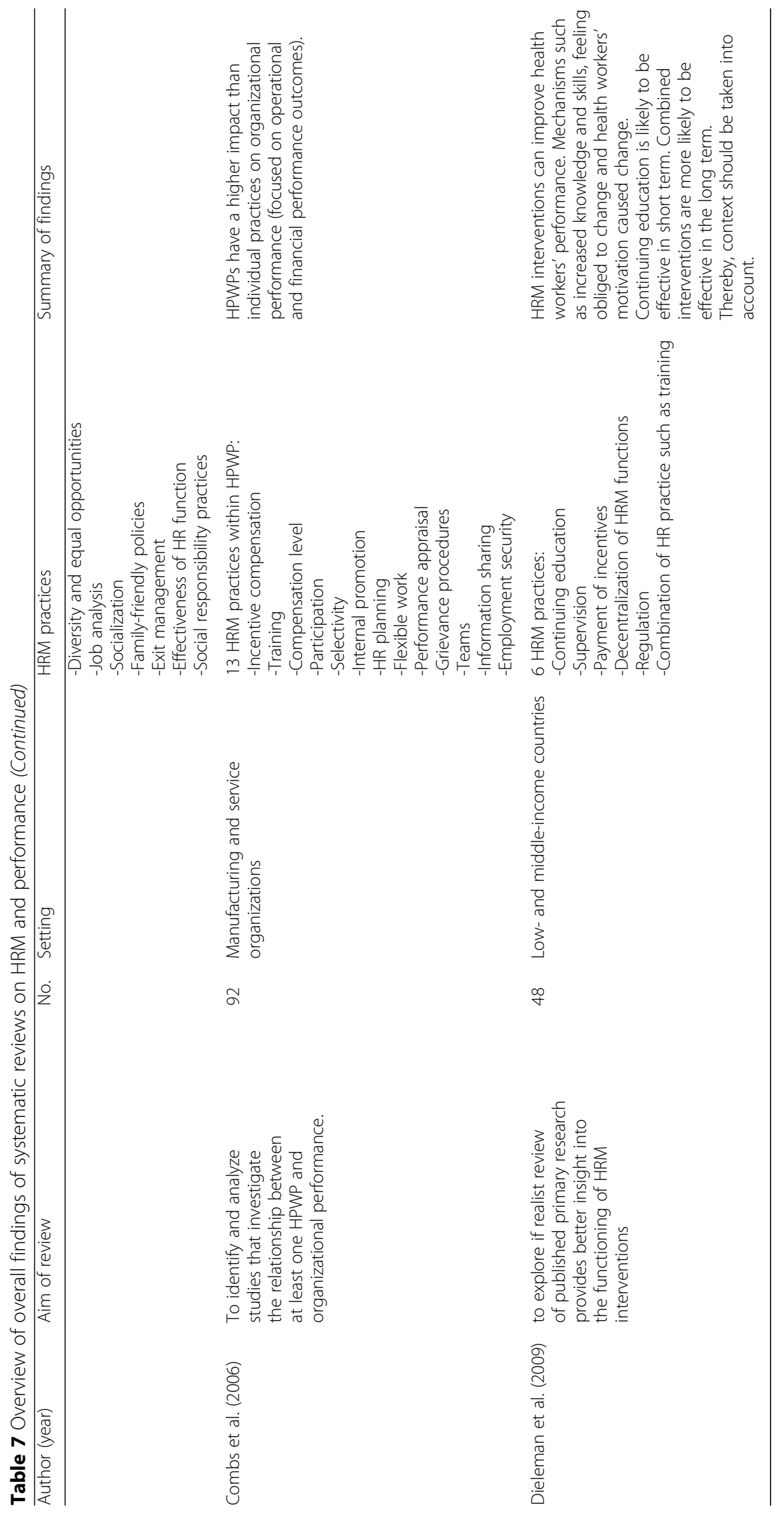


practices, which is believed to lead to superior outcomes in any setting [181]. Training is evidence to positively impact outcomes in all four performance categories. Training and educating caregivers in non-technical skills (e.g., communication, awareness, interaction) is a worldwide trend within the hospital setting and is proven to lead to higher team performance, patient safety, and organizational performance [181-183]. Task shifting/ role delegation in SSA hospitals is the second most researched single HRM practice, and mostly evidenced to relate to improved organizational and patient outcomes $[95,103,161,162,164]$. Task shifting is seen as one of the most important policy options to alleviate workforce shortage and skill mix imbalances in low-resource countries [184]. The most common task shifting, which requires leadership support, takes place in HIV treatment where tasks are delegated from doctors to nurses and other non-physician clinicians $[185,186]$.

Most included studies researched HRM bundles that included practices from multiple HRM domains: motivationenhancing, skills-enhancing, and empowerment-enhancing [28]. Motivation-enhancing practices were most frequently researched within HRM bundles, followed by skills-enhancing practices and empowerment-enhancing practices. A significant amount of studies provided evidences on the link between a HRM bundle and improved performance (e.g., $[16,17,19-21,24])$. Our findings show that an improvement in a specific outcome measure can be accomplished by different HRM practices or bundles and that similar HRM practices or bundles could enhance different outcome measures. For instance, job satisfaction could be improved through (a combination of) single HRM practices or bundles regarding training, management support, teamwork, promotion, autonomy, financial incentives, scheduling, and performance appraisal (e.g., [70, 79, 98, 109, 112, 117, 123, 127, 136]). This also holds for the outcomes; retention, motivation, and quality of care. Eight studies that examined a similar HRM bundles reported improvement in diverse outcome measures (e.g., employee performance, organizational outcome such as quality of care, efficiency, and patient satisfaction) $[81,82,87,91,137,176,178]$.

Previous studies have shown the importance of an internal fit within a HRM bundle, referring to an alignment between HRM practices [24, 26, 29, 187]. Notable is that several included studies have combined teamwork with individual financial incentives such as salary (e.g., $[99,101,105,129,135])$. Although this combination of HRM practices is in HRM literature often labelled as "deadly", financial incentives have shown to be effective and desirable in improvement programs in SSA [9, 34, 36, 37, 49-51]. This calls for further research.

\section{Limitations and future recommendations}

Our study included evidence on relationships between HRM practices and hospital performance in 19 SSA countries. Given the variety in results, we call for caution when generalizing the results to all SSA countries, or to health centers and clinics in SSA. Recognizing the importance of tailoring interventions to both internal and external context (also referred to as the "best fit approach" of HRM [27, 44, 45, 188]), we recommend future empirical research to report on relevant internal and external contextual factors. This will enable to build evidence on the mechanisms explaining how context and interventions together produce outcomes, as opposed to developing an evidence base for all of the different SSA contexts. Second, this review was restricted to peer-reviewed English articles and did not including books, grey literature, or any documents published in a foreign language. As a result, we may have failed to identify some evidence. Additionally, the inclusion criteria may have induced bias towards effective implementations and caused us to exclude interventions which produced little or adverse performance effects. Lastly, we note that our review produced little evidence on a direct relationship between HRM interventions and patient outcomes, or on outcomes at a team level. We recommend to conduct research in these areas, as team performance is evidenced to be particularly related to patient outcomes of hospitals $[189,190]$.

\section{Additional files}

Additional file 1: Search terms. (DOCX $16 \mathrm{~kb}$ )

Additional file 2: Number of studies that link a specific HRM practice to a specific outcome. (XLSX $11 \mathrm{~kb}$ )

Additional file 3: SSA countries represented in selected studies. (DOCX 14 kb)

\section{Acknowledgements}

The authors thank Wichor Bramer, who is a Librarian at the Erasmus Medical Center, Rotterdam, The Netherlands, for his vital contributions in executing the search strategy.

\section{Funding}

This research received no specific grant from any funding agency in the public, commercial, or not-for-profit sectors.

Availability of data and materials

All data are available at request.

Authors' contributions

The first author took the lead in writing the manuscript. The second and third authors revised and have co-written the manuscript. All authors

assessed the eligibility of the found studies. The first author summarized the selected full-text manuscripts.

\section{Ethics approval}

Not applicable

Consent for publication

Not applicable

Competing interests

The authors declare that they have no competing interests. 


\section{Publisher's Note}

Springer Nature remains neutral with regard to jurisdictional claims in published maps and institutional affiliations.

\section{Author details \\ 'Higher Education Institutions' Partnership, PO BOX 14051, Addis Ababa, Ethiopia. ${ }^{2}$ Erasmus School of Health Policy \& Management, Erasmus University Rotterdam, PO Box 1738, 3000 DR Rotterdam, The Netherlands. ${ }^{3}$ Prince Mohammad Bin Salman College (MBSC) of Business \& Entrepreneurship, 7082-BayLaSun-Juman St. Unit No. 1, King Abdullah Economic City 23964-2522, Kingdom of Saudi Arabia.}

Received: 2 May 2018 Accepted: 9 July 2018

Published online: 02 August 2018

\section{References}

1. Dolvo D. Wastage in the health workforce: some perspectives from African countries. Human Resources for Health. 2005:3:6

2. Chen C, Buch E, Wassermann T, Frehywot S, Mullan F, Omaswa F, et al. A survey of sub-Saharan African medical schools. Human Resources for Health. 2012;10:4.

3. Sartorius K, Sartorius BKD. A spatial model to quantify the mortality impact of service delivery in sub-Saharan Africa: an ecological design in utilizing data from South Africa. International Journal of Health Geographics. 2013:12:8.

4. Anyangwe SCE, Mtonga C. Inequalities in the Global Health Workforce: The Greatest Impediment to Health in sub-Saharan Africa. International Journal Environmental Research \& Public Health. 2007;4:2.

5. World Health Organization, Global Atlas of the Health Workforce, WHO Regional Office for Africa. Atlas of African Health Statistics 2016. African Health Observatory. http://www.aho.afro.who.int. Accessed 15 July 2017.

6. Wakaba M, Mbindyo P, Ochieng J, Kiriinya R, Todd J, Waudo A, et al. The public sector nursing workforce in Kenya: a country level analysis. Human Resources for Health. 2014;12:6.

7. Berhan $Y$, Berhan A. Reason for persistently high maternal and perinatal mortality in Ethiopia: Part I - Health System Factors. Ethiopian Journal of Health Sciences. 2014;24:119-36.

8. Naicker S, Plange-Rhule J, Tutt RC, Eastwood JB. Shortage of healthcare workers in developing countries-Africa. Ethnicity \& Disease. 2009;19

9. Hagoplan A, Thomson MJ, Fordyce M, Johnson KE, Hart LG. The migration of physicians from sub-Saharan Africa to the United States of America: measures of the African brain drain. Human Resources for Health. 2004:2:17.

10. Toure B, Avocksouma DA, Nyoni J, Ahmal A. Roadmap for scaling up human resources for improved health service delivery in the African region. WHO Africa Regional Office Brazzaville: Congo; 2012.

11. Murphy GP, Goma F, Mackenzie A. A scoping review of training and deployment policies for human resources for health for maternal, newborn, and child health in rural Africa. Human Resources for Health. 2014;13:27.

12. Bryan L, Conway M, Keesmaat T, McKenna S, Richardson B Strengthening sub-Saharan Africa's health system: a practical approach. McKinsey \& Company; 2010.

13. Institute for Health Metrics \& Evaluation (IHME), Health Development Network and The World Bank. The Global Burden of Disease: Generating Evidence, Guiding Policy-Sub Saharan Africa Regional Edition. Seattle, WA: IHME; 2013.

14. Derbew M, Lytin AD, Dicker RA. The surgical workforce shortage and successes in retaining surgical trainees in Ethiopia. Human Resources for Health. 2016;14:29.

15. Kebede D, Zielinski C, Mbondji EP, Sanou I, Kouvividila W, Lusamba-Dikassa PS. Human resources in health research institutions in sub-Saharan African countries: results of a questionnaire-based survey. Journal of the Royal Society of Medicine. 2014;107:85-95.

16. Dieleman $M$, Gerretsen B, van der Wilt GJ. Human resources management interventions to improve health workers' performance in low and middle income countries: a realist review. Health Research Policy and Systems. 2009;7:7.

17. Paauwe J. HRM and performance: achievements, methodological issues and prospects. Journal of Management Studies. 2009:46(1):129-42.

18. Rowe AK, Savigny DV, Lanata CF, Victora CG. How can we achieve and maintain high-quality performance of health workers in low-resource settings? The Lancet. 2005;366:1026-35.
19. Boselie P, Dietz G, Boon C. Commonalities and contradictions in HRM and performance research. Human Resource Management Journal. 2005; 15:67-94.

20. Combs J, Liu Y, Hall A, Ketchen D. How much do high-performance work practices matter? A meta-analysis of their effects on organizational performance. Personnel Psychology. 2006;59(3):501-28.

21. Vermeeren B, Steijn B, Tummer M, Poerstamper RJ, Sandra VB. HRM and its effect on employee, organizational and financial outcomes in health care organizations. Human Resources for Health. 2014;12:35.

22. Peccei R. Human resource management and the search for the happy workplace. Inaugural address. Rotterdam: Erasmus Research Institute of Management (ERIM); 2004.

23. West MA, Borris C, Dawson J, Scully J, Carter M, Anelay S, et al. The link between the management of employees and patient mortality in acute hopsitals. The International Journal of Human Resource Management. 2002; 13:1299-310.

24. West MA, Guthrie JP, Dawson JF, Borrill CS, Carter M. Reducing patient mortality in hospitals: the role of HRM. Journal of Organizational Behavior. 2006;27:983-1002

25. Kabene SM, Orchard C, Howard JM, Soliano MA, Leduc R. The importnce of human resouces management in health care : a global context. Human Resources for Health. 2006;4:20

26. Hyde P, Boaden R, Cortvriend P, Harris C, Marchington M, Pass S, et al. Improving Health through Human Resource Management. London: CIPD; 2008.

27. Harris $C$, Cortvriend $P$, Hydie $P$. Human resource management and performance in healthcare organizations. Journal of Health Organization and Management. 2007;21:448-59.

28. Subramony M. A meta-analytic investigation of the relationship between HRM bundles and firm performance. Human Resource Management. 2009; 48(5):745-68.

29. Boselie P, Chris B, Paauwe J. In search of balance- managing the dualities of HRM- an overview of the issues. Personnel Review. 2009;38(5):461-71.

30. Bach S, editor. Managing Human Resources: Personnel Management in Transition. England: John Wiley \& Sons; 2005.

31. Dieleman M, Harnmeijer JW. Improving health worker performance: in search of promising practices. Geneva: World Health Organization; 2006.

32. Buchan J. What difference does "good" HRM make? Human Resources for Health. 2004;2:6

33. Marchal B, Dedzo M, Kegels G. A realist evaluation of the magnitude of a well-performing regional hospital in Ghana. BMC Health Services Research. 2010;10:24.

34. Marchal B, Kegels G. Focusing on the software of managing health worker: what can we learn from high commitment management practices? International Journal of Health Planning Management. 2008;23(4):299-311.

35. Veld MF, Paauwe J, Boselie P. HRM and strategic climates in hospitals : does the message come across at the ward level. Human Resource Management Journal. 2010;20:339-56.

36. Mills EJ, Schabas WA, Volmink J. Walker R, Ford N, Katabira E, et al. Should active recruitment of health workers from sub-Saharan Africa be viewed as a crime? The Lancet. 2008:371,9613:685-688.

37. Willcox ML, Pearsman W, Daou P. Human resources for primary health care in sub-Saharan Africa: progress or stagnation. Human Resources for Health. 2015;13:76.

38. World Health Organization. Working together for health. World Health Report. Geneva: World Health Organization; 2006.

39. Boxall P, Ang SH, Bartram T. Analyzing the 'Black Box' of HRM: Uncovering HR goals, mediators, and outcomes in a standardized service environment. Journal of Manag Studies. 2011;48:1504-32.

40. Paauwe J, Boselie P. HRM and performance: what next? Human Resource Management Journal. 2005;15:68-82.

41. Nishi LH, Wright PM. Variability within organizations: Implications for strategic human resource management. In: Smith DB, editors. The people make the place: dynamic linkages between individuals and organizations. Taylor \& Francis Group; 2008. P. 225-248.

42. Pawson R, Tilley N. Realistic Evaluation London. 1997. Sage.

43. Van Aken JE. Management research based on the paradigm of the design sciences: The quest for field-tested and grounded technological rules. Journal of Management Studies. 2004;41:219-46.

44. Paauwe JHRM. Performance: achieving long term viability. New York: Oxford University Press; 2004. 
45. Veld M, Paauwe J, Boselie P. Mapping the context: Different scenarios for managing human resources in a changing hospital context. Rotterdam: Institute of Health Policy \& Management, Erasmus University Rotterdam; 2010.

46. Bradley S, Kamwendo F, Masanji H, de Pinho H, Waxman R, Boostrom C, et al. District managers' perceptions of supervision in Malawi and Tanzania. Human Resources for Health. 2013;11:43.

47. Van Lerberghe $W$, de Bethune $X$, van de Brouwere. Hospitals in subSaharan Africa: why we need more of what does not work as it should? Tropical Medicine \& International Health. 1997;2,8:799-808.

48. Cornell J, Zurn P, Stilwell B, Awases M, Braichet JM. Sub Saharan Africa: beyond the health worker migration crisis? Social Science \& Medicine. 2007; 64(9):1876-91.

49. Ferrinho P, Ferrinho P, Van Lerberghe W, Fronteira I, Hipólito F, Biscaia A. Dual practice in the health sector: review of the evidence. Human Resources for Health. 2004;2:14.

50. Lindelow M, Serneels $P$. The performance of health workers in Ethiopia: results from a qualitative research. Social Science \& Medicine. 2006;62(9):2225-35.

51. Habte D, Dussault G, Dovol D. Challenges confronting health workforce in sub-Saharan Africa. World Hospitals and Health Services. Journal of the International Hospital Federation. 2004;40:2.

52. Harries AD, Salaniponi FM. Improving health workers' performance in lowresource settings. The Lancet. 2005;366:5.

53. Centre for Reviews and Dissemination (CRD). Cochrane Guideline for Systematic Reviews: guidance for undertaking reviews in health care. University of York; 2008.

54. Higgins JPT, Green S, editors. Cochrane Handbook for Systematic Reviews of Interventions. England: John Wiley \& Sons; 2011.

55. Moher D, Liberati A, Tetzlaff J, Altman DG. Preferred reporting items for systematic reviews and meta-analyses: the PRISMA statement. International journal of surgery. 2010;8:336-41.

56. Al-Yaman F. United Nations Population Division, Department of Economic and Social Affairs. Journal of Population Research. 2004;4

57. Yule S, Flin R, Paterson-Brown S, Maran N. Non-technical skills for surgeons in the operating room: a review of the literature. Surgery. 2006;139:140-149.

58. Flin R, Yule S, Paterson-Brown S, Rowley D, Youngson G. Teaching surgeons about non-technical skills. Surgeon. 2007;5(2):86-9.

59. Systematic review resources: handbooks for doing systematic reviews. http://libguides.sph.uth.tmc.edu/systematic-review-guidance/SR-guides. Accessed 29 July 2016.

60. Mahdavi M, Malmstrom T, Van de Klundert JJ, Elkhuizen S, Vissers J. Generic operational models in health service operations management. A systematic review. Socio-Economic Planning Sciences. 2013;47:271-80.

61. Pluye P, Gagnon M, Griffiths F, Johnson-Lafleur J. A scoring system for appraising mixed methods research, and concomitantly appraising qualitative, quantitative and mixed methods primary studies in mixed studies reviews. International Journal of Nursing Studies. 2009;46:529-46.

62. Pace R, Pluye P, Bartlett G, Macaulay AC, Salsberg J, Jagosh J, et al. Testing the reliability and efficiency of the pilot Mixed Methods Appraisal Tool (MMAT) for systematic mixed studies review. International Journal of Nursing Studies. 2012;49:47-53.

63. Pluye P, Robert E, Cargo M, Bartlett G, O'Cathain A, Griffiths F, et al. Proposal: A mixed methods appraisal tool for systematic mixed studies reviews. Montréal: McGill University; 2011.

64. Amorin-Woods LG, LosCo BE. 'PICO-D Management'-a decision-aid for evidence-based chiropractice education and clinical practice. Journal of Chiropractice and manual Therapies. 2016;24:49.

65. Akhlaq A, McKinstry B, Muhammad KB, Sheikh A. Barriers and facilitators to health information exchange in low- and middle-income country settings: a systematic review. Health Policy and Planning. 2016;31:1310-20.

66. Van Panhuis WG, Paul P, Emerson C, Grefenstette J, Wilder R, Herbst AJ, et al. A systematic review of barriers to data sharing in public health. BMC Public Health. 2014;14:1144.

67. Humphries S, Stafinski T, Mumtaz Z, Menon D. Barriers and facilitators to evidence-use in program management: a systematic review of the literature. BMC Health Services Research. 2014;14:171

68. Ajemigbitse AA, Omole MK, Ezike NC, Erhun WO. Assessment of the knowledge and attitudes of intern doctors to medication prescribing errors in a Nigeria tertiary hospital. Journal of Basic and Clinical Pharmacy. 2013;5:1.

69. Aberese-Ako M, van Dijk H, Arhinful DK, Agyepong IA. Your health our concern, our health whose concern?: perceptions of injustice in organizational relationships and processes and frontline health worker motivation in Ghana. Health Policy and Planning. 2014;29:15-28.

70. Ackerman ER, Bezuidenhout MC. Staff dissatisfaction in the theatre complex of a private hospital. Curationis. 2007;30:68-73.

71. Francis A, Adzei Roger A. Motivation and retention of health workers in Ghana's district hospitals. Journal of Health Organization and Management. 2012;26:467-85.

72. Simiyu AN, Moronge M. Influence of human resource functions on employee performance in the developed health sector in Kenya: a case of Mbagathi District hospital. The Strategic Journal of Business \& Change Management. 2015;2:2099-136.

73. Allegranzi B, Sax H, Bengaly L, Richet H, Minta DK, Chraiti MN, et al. Successful implementation of the World Health Organization Hand Hygiene Improvement Strategy in a Referral Hospital in Mali, Africa. Infection Control \& Hospital Epidemiology. 2010;31:133-141.

74. Akinyemi $\mathrm{O}$, Atilola $\mathrm{O}$. Nigerian resident doctors on strike: insights from and policy implications of job satisfaction among resident doctors in a Nigerian teaching hospital. International Journal of Health Planning and Management. 2013;28:46-61.

75. Ajayi NA. Impact of health informatics on nurses' computer skills and the role of the library. The Electronic Library. 2013;31:157-66.

76. Abubakar R, Chauhan A, Kura K. Relationship between perceived organizational politics, organizational trust, human resource management practices and turnover intention among Nigerian nurses. Malaysia: Universiti Utara Malaysia; 2014.

77. Asegid A, Belachew T, Yimam E. Factors influencing job satisfaction and anticipated turnover among nurses in Sidama Zone Public Health Facilities. South Ethiopia. Nursing Research and Practice. 2014;

78. Ackerman ER, Bezuidenhout MC. Staff dissatisfaction in the theatre complex of a private hospital. Curationis. 2007;30:3.

79. Ashmore J. Going private': a qualitative comparison of medical specialists' job satisfaction in the public and private sectors of South Africa. Human Resources for Health. 2013;11:1.

80. Ashmore J, Gilson L. Conceptualizing the impacts of dual practice on the retention of public sector specialists-evidence from South Africa. Human Resources for Health. 2015;13:3.

81. Atambo WN, Kabare K, Munene C, Nyamwamu WB. Enhancing the role of employee recognition towards improving performance: A survey of Kenyatta National Hospital. Kenya. International Journal of Arts and Commerce. 2012;1:95-108.

82. Atambo WN, Kabare K, Munene C, Mayogi N. Employee recognition and training on performance: a survey of public hospitals in Kenya. Global Business and Economics Research Journal. 2013;2:29-44.

83. Atambo WN, Kabare K, Munene $C$. The role of employee incentives on performance: a survey of public hospitals in Kenya. Global Business and Economics Research Journal. 2013;2:29-44.

84. Aveling EL, Kayonga Y, Nega A, Dixon-Woods M. Why is patient safety so hard in low-income countries? A qualitative study of healthcare workers' views in two African hospitals. Globalization and Health. 2015;11:6-14.

85. Awasses $\mathrm{MH}$, Bezuidenhout MC, Roos JH. Factors affecting the performance of professional nurses in Namibia. Curationis. 2013;6:1-8.

86. Ayieko P, Ntoburi S, Wagai J, Opondo C, Opiyo N, Migiro S, et al. A multifaceted intervention to implement guidelines and improve admission paediatric care in Kenyan district hospitals: a cluster randomised trial. PLoS Med. 2011:8:4.

87. Waju B, Jira C, Sudhakar M. Assessment of quality of health care in Jimma Zone. Southwest Ethiopia. Ethiopian Journal of Health Sciences. 2011;21

88. Bhengu BR. Exploring the critical care nurses' experiences regarding moonlighting. Curationis. 2001;24:48-53.

89. Bradley E, Hartwig K, Rowe LA, Cherlin EJ,Pashman J, Wong R, et al. Hospital quality improvement in Ethiopia: a partnership-mentoring model. International Journal for Quality in Health Care. 2008:20:392-399.

90. Bradley S, McAuliffe E. Mid-level providers in emergency obstetric and newborn health care: factors affecting their performance and retention within the Malawian health system. Human Resources for Health. 2009;7:14.

91. Dagne T, Beyene W, Berhanu N. Motivation and factors affecting it among health professionals in the public hospitals. Central Ethiopia. Ethiopian Journal of Health Sciences. 2015:25:231-42.

92. De Brouwere V, Dieng T, Diadhiou M, Witter S, Denerville W. Task shifting for emergency obstetric surgery in district hospitals in Senegal. Reproductive Health Matters. 2009;17:32-44. 
93. Dieleman M, Toonen J, Touré H, Martineau T. The match between motivation and performance management of health sector workers in Mali. Human Resources for Health. 2006;4:2.

94. Eygelaar J, Stellenbeg EL. Barriers to quality patient care in rural district hospitals. Curationis. 2012;35:1-8.

95. Ferrinho P, Sidat M, Goma F, Dussault G. Task-shifting: experiences and opinions of health workers in Mozambique and Zambia. Human Resources for Health. 2012;10:34

96. Galukande M, Kaggwa S, Sekimpi P, Kakaire O, Katamba A, Munabi I, et al. Use of surgical task shifting to scale up essential surgical services: a feasibility analysis at facility level in Uganda. BMC Health Services Research. 2013;13:292.

97. Hall EJ. Nursing attrition and the work environment in South African health facilities. Curationis. 2004;

98. Honda A, Vio F. Incentives for non-physician health professionals to work in the rural and remote areas of Mozambique-a discrete choice experiment for eliciting job preferences. Human Resources for Health. 2015;13:23.

99. Ibeziako OJ, Chabikuli ON, Olorunju S. Hospital reform and staff morale in South Africa: a case study of Dr Yusuf Dadoo Hospital. South African Family Practice. 2013;55:180-5.

100. Issahaku A, Ahmed D, Bewa-Erinibe PL. Enhancing employee performance through training: the case of Tamale Teaching Hospital in Ghana. European Journal of Business and Management. 2014;6:29.

101. Jack H, Canavan M, Ofori-Atta A, Taylor L, Recruitment BE. Retention of mental health workers in Ghana. PLoS ONE. 2013;8:e57940.

102. Jacobs E, Roodt G. Organisational culture of hospitals to predict turnover intentions of professional nurses. Health SA Gesondheid. 2008;13:1.

103. Jennings L, Yebadokpo AS, Affo J, Agbogbe M, Tankoano A. Task shifting in maternal and newborn care: a non-inferiority study examining delegation of antenatal counseling to lay nurse aides supported by job aids in Benin. Implementation Science. 2011;6:2.

104. Kamanzi J, Nkosi Z. Motivation levels among nurses working at Butare University Teaching Hospital, Rwanda. African Africa Journal of Nursing and Midwifery. 2011;13:2.

105. Kekana HP, du Rand EA, van Wyk NC. Job satisfaction of registered nurses in a community hospital in the Limpopo Province in South Africa. Curationis. 2007;30:24-35

106. Khamis K, Njau B. Health care worker's perception about the quality of health care at the outpatient department in Mwananyamala Hospital in Dar es Salaam. Tanzania. Tanzania Journal of Health Research. 2016;18:1.

107. Kotzee K, Couper ID. What interventions do South African qualified doctors think will retain them in rural hospitals of the Limpopo province of South Africa? Journal of Rural and Remote Health. 2006;6:581.

108. Kruger A, Bezuidenhout C. Factors influencing female doctors' career decisions at Tshwane District Hospital, Pretoria. South African Family Practice. 2015;57:12-7.

109. Lephoko CSP, Bezuidenhout MC, Roos JH. Organizational climate as a cause of job dissatisfaction among nursing staff in selected hospitals within the Mpumalanga Province. Curationis. 2006;29:28-36

110. Leshabari MT, Muhondwa EP, Mwangu MA, Mbembati NA. Motivation of health care workers in Tanzania: a case study of Muhimbili National Hospital. East African Journal of Public Health. 2008;5:32.

111. Longmore $B$, Ronnie L. Human resource management practices in a medical complex in the Eastern Cape, South Africa: assessing their impact on the retention of doctors. South African Medical Journal. 2014;104:368-71.

112. Luboga S, Hagopian A, Ndiku J, Bancroft E, McQuide P. Satisfaction, motivation, and intent to stay among Ugandan physicians: a survey from 18 national hospitals. International Journal of Health Planning and Management. 2011;26:2-17.

113. Makapela NC, Useh U. Rural allowance and the retention of health professionals in selected hospitals in the North West Province of South Africa. Journal of Human Ecology. 2013;44:129-38.

114. Mathauer I, Imhoff I. Health worker motivation in Africa: the role of nonfinancial incentives and human resource management tools. Human Resources for Health. 2006:4:24

115. Mbindyo P, Gilson L, Blaauw D, English M. Contextual influences on health worker motivation in district hospitals in Kenya. Implementation Science. 2009;4:43.

116. McAuliffe E, Bowie C, Manafa O, Maseko F, MacLachlan M, Hevey D, et al. Measuring and managing the work environment of the mid-level provider-the neglected human resource. Human Resources for Health 2009;7:13.

117. McAuliffe E, Daly M, Kamwendo F, Masanja H, Sidat M, de Pinho H. The critical role of supervision in retaining staff in obstetric services: a three country study. PLOS ONE. 2013;8:e58415.

118. Mokoka E, Oosthuizen MJ, Ehlers VJ. Retaining professional nurses in South Africa: nurse managers' perspectives. Health SA Gesondheid. 2010;15:484.

119. Mubyazi GM, Bloch P, Byskov J, Magnussen P, Bygbjerg LC, Hansen KS. Supply-related drivers of staff motivation for providing intermittent preventive treatment of malaria during pregnancy in Tanzania: evidence from two rural districts. BMC. Malaria Journal. 2012;11:48

120. Nwude CE, Uduji Jl. An exploratory study of the relationship of workforce compensation and job performance in the federal teaching hospitals in Nigeria. Journal of Industrial Engineers. 2013;3:3.

121. Nyathi M, Jooste K. Working conditions that contribute to absenteeism among nurses in a provincial hospital in the Limpopo Province. Curationis. 2008;31:28-37.

122. Osissom HE, Nzewi HN, Ifechi IL. Flexible working hours and employee performance in selected hospitals in Awka Metropolis, Anambra State, Nigeria. International Journal of Human Resource Management and Research. 2015:5:1-10.

123. Pietersen C. Job satisfaction of hospital nursing staff. South African Journal of Human Resource Management. 2005:3:19-25.

124. Pillay R. Work satisfaction of professional nurses in South Africa: a comparative analysis of the public and private sectors. Human Resources for Health. 2009;7:15.

125. Prytherch H, Kakoko DCV, Leshabari MT, Sauerborn R, Marx M. Maternal and newborn healthcare providers in rural Tanzania: in-depth interviews exploring influences on motivation performance and job satisfaction. Rural and Remote Health. 2012;12:2072.

126. Rispel LC, Chirwa T, Blaauw D. Transforming nursing in South Africa: does moonlighting influence South African nurses' intention to leave their primary jobs? Global Health Action. 2014;7:25754.

127. Selebi C, Minnaar A. Job satisfaction among nurses in a public hospital in Gauteng "It is all about salaries". Curationis. 2007;30:3.

128. Sikwese A, Mwape L, Mwanza J, Kapungwe A, Kakuma R, Imasiku M, et al. Human resource challenges facing Zambia's mental health care system and possible solutions: results from a combined quantitative and qualitative study. International Review of Psychiatry. 2010;22:550-557.

129. Siril H, Hirschhorn LR, Hawkins C, Garcia ME, Li MS, Ismail S, et al. Stress, Motivation, and Professional Satisfaction among health care workers in HIV care and treatment centers in Urban Tanzania. A cross-sectional study. East African Journal Public Health. 2011;8:17-24.

130. Ssengoba F, Atuyambe L, McPake B, Hanson K, Okuonzi S. What could be achieved with greater public hospital autonomy? Comparison of Public and PNFP Hospitals in Uganda. Public Administration and Development. 2002; 22:415-28.

131. Stodel JM, Stewart-Smith A. The influence of burnout on skills retention of junior doctors at Red Cross War Memorial Children's Hospital: a case study. South African Medical Journal. 2011;101:115-8

132. Tabatabai P, Prytherch H, Baumgarten I, Kisanga OME, Schmidt-Ehry B, Marx $M$. The internal migration between public and faith-based health providers: a cross-sectional, retrospective and multicenter study from southern Tanzania. Tropical Medicine and International Health. 2013;18:887-97.

133. Thatte N, Choi Y. Does human resource management improve family planning service quality? Analysis from the Kenya Service Provision Assessment 2010. Health Policy and Planning. 2015;30:356-67.

134. Uwaliraye P, Puoane T, Binagwaho A, Basinga P. Factors influencing the job performance of nurses and midwives in postpartum units in two district hospitals in Rwanda. Africa Journal of Nursing and Midwifery. 2013;15:59-69.

135. Yami 'A, Hamza L, Hassen A, Jira C, Sudhakar M. Job satisfaction and its determinants among health workers in Jimma University Specialized Hospital, Southwest Ethiopia. Ethiop Journal of Health Sciences. 2011;21:19-27

136. Bekker M, Coetzee S, Klopper H, Ellis SM. Non-nursing tasks, nursing tasks left undone and job satisfaction among professional nurses in South African hospitals. Journal of Nursing Management. 2015;23:1115-25.

137. Chandler Cl, Chonya S, Mtei F, Reyburn H, Whitty CJ. Motivation, money and respect: a mixed-method study of Tanzanian non-physician clinicians. Social science \& medicine. 2009;68:2078-88. 
138. Chi PC, Bulage P, Urdal H, Sundby J. Barriers in the delivery of emergency obstetric and neonatal care in post-conflict Africa: qualitative case studies of Burundi and Northern Uganda. PLoS ONE. 2015;10:9.

139. Chirwa M. What specific problems do nurse managers in Malawi report they experience in ensuring quality care. Africa Journal of Nursing and Midwifery. 2000;2:7-11.

140. Hollup O. Nurses in Mauritius motivated by extrinsic rewards: a qualitative study of factors determining recruitment and career choices. International Journal of Nursing Studies. 2012;49:1291-8.

141. Klopper H, Coetzee S, Pretorius R, Bester P. Practice environment, job satisfaction and burnout of critical care nurses in South Africa. Journal of Nursing Management. 2012;20:685-95.

142. Lasebikan VO, Oyetunde MO. Burnout among Nurses in a Nigerian General Hospital: prevalence and associated factors. International Scholarly Research Network. 2012:1-6.

143. Mudaly P, Nkosi ZZ. Factors influencing nurse absenteeism in a general hospital in Durban. South Africa. Journal of Nursing Management. 2015;23: 623-31

144. Tibandebage $P$, Kida T, Mackintosh M, Ikingura J. Can managers empower nurse-midwives to improve maternal health care? A comparison of two resource-poor hospitals in Tanzania. International Journal of Health Planning and Management. 2016;31:379-95.

145. Courtright P, Ndegwa L, Msosa J, Banzi J. Use of our existing eye care human resources: assessment of the productivity of cataract surgeons trained in Eastern Africa. Archives of Ophthalmology. 2007; 125:684-7.

146. Bergman S, Deckelbaum D, Lett B, Haas B, Demyttenaere S, Munthali V, et al. Assessing the impact of the trauma team training program in Tanzania. Journal of Trauma and Acute Care Surgery. 2008;65:879-883.

147. Doherty J, Conco D, Couper I, Fonn S. Developing a new mid-level health worker: lessons from South Africa's experience with clinical associates. Global Health Action. 2013;6:19282.

148. Esan O, Adeoye A, Onakoya P, Opeodu O, Owonikoko K, Olulana D, et al. Features of residency training and psychological distress among residents in a Nigerian teaching hospital. South African Journal of Psychology. 2014;20:46-50.

149. Kamau JK, Omondi M. Relationship between human resource management practices and staff retention in public hospitals in Kenya: a case study of Gatundu District Hospital. The strategic Journal of Business and Change Management. 2015;2:1775-98.

150. Letlape HR, Koen MP, Coetzee SK, Koen V. The exploration of in-service training needs of psychiatric nurses. Health SA Gesondheid. 2014;19:63.

151. Madzimbamuto FD, Ray SC, Mogobe KD, Ramogola-Masire D, Phillips R, Haverkamp M, et al. A root-cause analysis of maternal deaths in Botswana: towards developing a culture of patient safety and quality improvement. BMC Pregnancy and Childbirth. 2014;14:231.

152. Mahlo S, Muller M. Problems experienced by role players within the managed healthcare context in Gauteng. Curationis. 2000;23:37-41.

153. Manongi RN, Nasuwa FR, Mwangi $R$, Reyburn H, Poulsen A, Chanler CR. Conflicting priorities: evaluation of an intervention to improve nurseparent relationships on a Tanzanian pediatric ward. Human Resources for Health. 2009;7:50.

154. Mclntosh J, Stellenberg EL. Effect of a staffing strategy based on voluntary increase in working hours on quality of patient care in a hospital in KwaZulu-Natal. Curationis. 2009;32:11-20.

155. Mdumaa E, Ersdal H, Svensen E, Kidantoh, Auestad B, Perlman JFrequent brief on-site simulation training and reduction in 24-h neonatal mortality-An educational intervention study. Resuscitation. 2015;93:1-7.

156. Nabirye RC. Occupational stress, job satisfaction, and job performance among hospital nurses in Kampala Uganda. Birmingham: University of Alabama at Birmingham; 2010.

157. Ndetei DM, Khasakhala L, Omolo JO. Incentives for health worker retention in Kenya: an assessment of current practice. African Mental Health Foundation, University of Namibia. In: University of Limpopo and ECSARegional Health Community; 2008.

158. Nigussie N, Demissie A. Relationship between leadership styles of nurse managers and nurses' job satisfaction in Jimma University Specialized Hospitals. Ethiopian Journal of Health Sciences. 2013;23:1.

159. Okeke JO. Shortage of health professionals: a study of recruitment and retention factors that impact rural hospitals in Lagos State, Nigeria. In: University of Phoenix; 2008.
160. Okurame DE. Mentoring and organizational constraints as predictors of attitudes to work in the Nigerian public health sector. Journal of health and human services administration. 2009:342-71.

161. Olson D, Preidis GA, Milazi R, Spinler JK, Lufesi N, Mwansambo C, et al. Task shifting an inpatient triage, assessment and treatment programme improves the quality of care for hospitalized Malawian children. Tropical Medicine and International Health. 2013;18:879-886.

162. O'Malley G, Asrat L, Sharma A, Hamunime N, Stephanus Y, Brandt L, et al. Nurse task shifting for antiretroviral treatment services in namibia: implementation research to move evidence into action. PLOS ONE. 2014;9,3:92014.

163. Rauf W, Blitz JJ, Geyser M, Rauf A. Quality improvement cycles that reduced waiting times at Tshwane District Hospital Emergency Department. South African Family Practice. 2008;50:43.

164. Sanjana P, Torpey K, Schwarzwalder A, Simumba C, Kasonde P, Nyirenda L, et al. Task-shifting HIV counseling and testing services in Zambia: the role of lay counselors. Human Resources for Health. 2009;7:44.

165. Thomas LS, Valli A. Levels of occupational stress in doctors working in a South African public-sector hospital. SAMJ. 2006;96:11.

166. Uys LR, Minnaar A, Simpson B, Reid S. The effect of two models of supervision on selected outcomes. Journal of Nursing Scholarship. 2005;37:282-8.

167. Yboah MA, Ansong MO, Yiranbon HAA, Gyebil FA. Determinants of workplace stress among healthcare professionals in Ghana: an empirical analysis. International Journal of Business and Social Science. 2014;5:4.

168. Rawlins B, Garrison K, Brechin SJG, Njeru A, Rakuom CP. High-performing reproductive healthcare facilities in Kenya: why they exceed expectations. Baltimore: United States Agency for International. Development. 2003;

169. Giuseppe R, Machoki MI, Mwalkambo, Good MJVHIV, Disease plague, demoralization and "Burnout": resident experience of the medical profession in Nairobi, Kenya. Culture, Medicine and Psychiatry. 2002; 26:55-86.

170. Ngao MD. Assessing Barriers to implementation of nursing process among nurses working at Machakos level 5 Hospital. Nairobi: University of Nairobi; 2013.

171. Kotagal M, Lee P, Habiyakare C, Dusable R, Kanama P. Improving quality in resource poor settings: observational study from rural Rwanda. BMJ. 2009;339: $1311-3$

172. Crofts JF, Mukuli T, Murove BT, Ngwenya S, Mhlanga S, Dube M, et al. Onsite training of doctors, midwives and nurses in obstetric emergencies, Zimbabwe. Bulletin World Health Organization. 2015;93:347-351.

173. Downing J, Batuli M, Kivumbi G, Kabahweza J, Grant L, Murray SA, et al. A palliative care link nurse programme in Mulago Hospital, Uganda: an evaluation using mixed methods. BMC Palliative Care. 2016;15:40.

174. Faye A, Fournier, Diop I, Philibert A, Florence Morestin F, Dumont ADeveloping a tool to measure satisfaction among health professionals in sub-Saharan Africa. Human Resources for Health. 2013;11:30.

175. Van der Doef M, Mbazzi FB, Verhoeven C. Job conditions, job satisfaction, somatic complaints and burnout among East African nurses. Journal of Clinical Nursing. 2012;21:1763-75.

176. Srofenyoh E, Ivester T, Engmann C, Olufolabi A, Bookman L, Owen M. Advancing obstetric and neonatal care in a regional hospital in Ghana via continuous quality improvement. International Journal of Gynecology and Obstetrics. 2012;116:17-21.

177. Woldegabriel Z, Ejigu Y, Weldegebreal F, Woldie M. Motivation of health workers and associated factors in public hospitals of West Amhara. Northwest Ethiopia. Patient Preference and Adherence. 2016;10:159-69.

178. Puoane T, Cuming K, Sanders D, Ashworth A. Why do some hospitals achieve better care of severely malnourished children than others? Five-year follow-up of rural hospitals in Eastern Cape. South Africa. Health Policy and Planning. 2008;23:428-37.

179. Batterham RW, Buchbinder R, Beauchamp A, Dodson S, Elsworth GR, Osborne RH. The OPtimising HEalth LIterAcy (Ophelia) process: study protocol for using health literacy profiling and community engagement to create and implement health reform. BMC Public Health. 2014;14:694.

180. Pawson R, Greenhalgh T, Harvey G, Walshe K. Realist review-a new method of systematic review designed for complex policy interventions. J Health Serv Res Policy. 2005;10:21-34.

181. Pfeffer J. Seven Practices of successful organizations. California Management Review. 1998:40:96-124.

182. Weaver SJ, Lyons R, DiazGranados D, Michael AR, Eduardo S, James O, et al. The anatomy of health care team training and the state of practice: a critical review. Academic Medicine. 2010;85:1746-1760. 
183. McCulloch P, Rathbone J, Catchpole K. Interventions to improve teamwork and communications among healthcare staff. Systematic review. British Journal of Surgery. 2011;98:469-79.

184. Buljac-Samardzic M, Dekker-van Doorn CM, van Wijngaarden JDH, van Wijk KP. Interventions to improve team effectiveness: a systematic review. Health Policy. 2010;94,3:183-95.

185. Fulton BD, Scheffler RM, Sparkes SP, Auh EY, Vujicic M, Soucat A. Health workforce skill mix and task shifting in low income countries: a review of recent evidence. Human Resources for Health. 2011;9:1.

186. Lehmann U, Van Damme W, Barten F, Sanders D. Task shifting: the answer to the human resources crisis in Africa? Human Resources for Health. 2009;7:49.

187. Callaghan M, Ford N, Schneider HA. Systematic review of taskshifting for HIV treatment and care in Africa. Human Resources for Health. 2010;8:8.

188. Corine B. HRM and Fit: Survival of the fittest?? Rotterdam: Erasmus University Rotterdam, ERIM; 2008.

189. Boet S, Bould D, Fung L, Qosa H, Perrier L, Tavares WR, Reeves S, Tricco AC. Transfer of learning and patient outcome in simulated crisis resource management: a systematic review. Canadian Journal of Anesthesia. 2014;61:571-82.

190. Fung L, Boet S, Bould M, Dylan QH, Perrier L, Tricco A, et al. Impact of crisis resource management simulation-based training for interprofessional and interdisciplinary teams: A systematic review, Journal of Interprofessional Care. 2015;29:433-444.

Ready to submit your research? Choose BMC and benefit from:

- fast, convenient online submission

- thorough peer review by experienced researchers in your field

- rapid publication on acceptance

- support for research data, including large and complex data types

- gold Open Access which fosters wider collaboration and increased citations

- maximum visibility for your research: over $100 \mathrm{M}$ website views per year

At $\mathrm{BMC}$, research is always in progress.

Learn more biomedcentral.com/submissions 